\title{
Smad3-Targeted Therapy Protects against Cisplatin-Induced AKI by Attenuating Programmed Cell Death and Inflammation via a NOX4-Dependent Mechanism
}

\author{
Qin Yang ${ }^{\mathrm{a}} \mathrm{Li} \mathrm{Gao}^{\mathrm{a}}$ Xiao-wei Hu ${ }^{\mathrm{a}}$ Jia-nan Wang ${ }^{\mathrm{a}}$ Yao Zhang ${ }^{\mathrm{a}}$ \\ Yu-hang Dong ${ }^{a}$ Hui Yao Lan ${ }^{b}$ Xiao-ming Meng ${ }^{a}$ \\ a Inflammation and Immune Mediated Diseases Laboratory of Anhui Province, Anhui Institute of Innovative Drugs, \\ School of Pharmacy, Anhui Medical University, The Key Laboratory of Anti-inflammatory and Immune Medicines, \\ Ministry of Education, Hefei, China; bepartment of Medicine \& Therapeutics, Li Ka Shing Institute of \\ Health Sciences, and Shenzhen Research Institute, The Chinese University of Hong Kong, Hong Kong, China
}

\section{Keywords}

Acute kidney injury $\cdot$ Cisplatin · Smad3 · Nox4 ·

Programmed cell death $\cdot$ Inflammation

\begin{abstract}
Background: Transforming growth factor- $\beta$ (TGF- $\beta$ )/Smad signaling is the central mediator in renal fibrosis, yet its functional role in acute kidney injury (AKI) is not fully understood. Recent evidence showed that TGF- $\beta /$ Smad3 may be involved in the pathogenesis of $\mathrm{AKI}$, but its functional role and mechanism of action in cisplatin-induced AKI are unclear. Objectives: Demonstrating that Smad3 may play certain roles in cisplatin nephropathy due to its potential effect on programmed cell death and inflammation. Methods: Here, we established a cisplatin-induced AKI mouse model with Smad3 knockout mice and created stable in vitro models with Smad3 knockdown tubular epithelial cells. In addition, we tested the potential of Smad3-targeted therapy using 2 in vivo protocols - lentivirus-mediated Smad3 silencing in
\end{abstract}

vivo and use of naringenin, a monomer used in traditional Chinese medicine and a natural inhibitor of Smad3. Results: Disruption of Smad3 attenuated cisplatin-induced kidney injury, inflammation, and NADPH oxidase 4-dependent oxidative stress. We found that Smad3-targeted therapy protected against loss of renal function and alleviated apoptosis, RIPK-mediated necroptosis, renal inflammation, and oxidative stress in cisplatin nephropathy. Conclusions: These findings show that Smad3 promotes cisplatin-induced AKI and Smad3-targeted therapy protects against this pathological process. These findings have substantial clinical relevance, as they suggest a therapeutic target for AKI.

(C) 2021 The Author(s)

Published by S. Karger AG, Basel

\section{Introduction}

Acute kidney injury (AKI) is a common clinical entity with high morbidity and mortality. AKI is induced by hemodynamic, hypoxic, mechanical, and toxic agents $[1,2]$.

Q.Y., L.G., and X.-w.H. contributed equally.

karger@karger.com www.karger.com/kdd

Karger $\stackrel{\text { ' }}{5}$

BOPEN ACCESS
(C) 2021 The Author(s)

Published by S. Karger AG, Basel

This article is licensed under the Creative Commons AttributionNonCommercial-NoDerivatives 4.0 International License (CC BYNC-ND) (http://www.karger.com/Services/OpenAccessLicense) Usage and distribution for commercial purposes as well as any distribution of modified material requires written permission.
Xiao-ming Meng

School of Pharmacy, Anhui Medical University

Mei Shan Road No. 81, Hefei 230032 (China)

mengxiaoming@ahmu.edu.cn

Hui-yao Lan

Department of Medicine \& Therapeutics

Li Ka Shing Institute of Health Sciences and Shenzhen Research Institute

The Chinese University of Hong Kong, Hong Kong 999077 (China)

hylan@ cuhk.edu.hk 
Although the common pathological features of AKI include programmed cell death and excessive inflammation, its detailed mechanism and effective therapy need to be explored further.

Transforming growth factor- $\beta$ (TGF- $\beta$ ) functions by activating downstream canonical and noncanonical pathways and regulating multiple biological processes including cell growth, differentiation, and immune response. The function of the TGF- $\beta /$ Smad signaling system has been extensively studied in CKD $[3,4]$. Although the role of TGF- $\beta$ in AKI has drawn significant attention recently, it is still controversial [5]. Previous studies reported that TGF- $\beta 1$ may be protective in AKI because blockade of TGF- $\beta 1$ in mice accelerated kidney injury and loss of renal function [6-8]. However, other studies found that depletion of TGF- $\beta$ receptor attenuated, whereas its activation promoted, AKI progression $[9,10]$. This was further confirmed by a recent study showing that TGF- $\beta$ promoted colistin-induced AKI [11]. Therefore, it is necessary to further explore the functional role of downstream Smads in different disease conditions. Smad2 and Smad3 are pivotal downstream effectors in renal fibrosis [1215]. Knockout (KO) of Smad 3 attenuates, but conditional KO of Smad2 promotes, renal fibrosis in UUO nephropathy $[12,16]$. However, our group recently found that unlike its protective role in renal fibrosis, Smad2 promoted AKI by inducing programmed cell death and inflammation, even though Smad 2 and Smad3 are both highly activated in cisplatin-induced AKI model [17].

The TGF- $\beta /$ Smad 3 signaling pathway plays an important role in various kidney diseases. Previous studies showed that $S$ mad3 is an important mediator of glomerulosclerosis and renal interstitial fibrosis [18]. Smad3 deficiency prevents renal dysfunction, renal fibrosis, and inflammation in type 2 diabetic nephropathy [19], and combined blockade of Smad3 and JNK pathways ameliorates progressive fibrosis in folic acid nephropathy [20]. Additionally, $\mathrm{KO}$ of Smad3 attenuated renal damage by limiting renal inflammation in a murine model of ischemic AKI [21], and C-reactive protein accelerated AKI by suppressing CDK2/cyclin E in Smad3-dependent mechanisms [22]; these results were further supported by our recent finding that $S m a d 3$ promotes AKI sensitivity in diabetic mice [23]. However, the functional role of Smad3 and its mechanism of action in cisplatin-induced AKI have not been studied. More importantly, it is not clear whether Smad3-targeted therapy can be used to treat kidney injury.

In this study, we generated Smad3 KO mice and Smad3 knockdown (KD) cell lines, the function of Smad3 in cis- platin-induced oxidative stress, programmed cell death, and inflammation was tested both in vivo and in vitro. We then evaluated 2 protocols of Smad3-targeted therapies in a cisplatin-induced AKI model. One protocol involved using lentivirus-mediated Smad3 silencing in vivo, and the other involved treatment of mice with a natural Smad3 inhibitor, naringenin, a flavonoid from grapefruit and citrus fruits that is known to have antiinflammatory properties [24-26]. Our findings reveal the function and mechanism of Smad3 in cisplatin-induced $\mathrm{AKI}$ and provide evidence for $\mathrm{Smad} 3$ as a promising therapeutic target in AKI.

\section{Methods}

\section{Reagents}

Cisplatin was obtained from Sigma-Aldrich (Sigma, San Diego, CA, USA). Naringenin was obtained from Shanxi Huike Botanical Development Co., Ltd. (Xian, Shanxi, China). Annexin V-FITC/ PI Apoptosis Detection Kit was purchased from Beyotime (Shanghai, China). Periodic acid-Schiff (PAS), Creatinine Assay Kit, and BUN Assay Kit were obtained from Nanjing Jiancheng Bioengineering Institute (Nanjing, China). Fetal bovine serum (FBS), DMEM, and other cell culture reagents were purchased from Invitrogen. Antibodies specific to KIM1 and $\beta$-actin were purchased from Santa Cruz Biotechnology (Santa Cruz, CA, USA). RIPK1 and RIPK3 were obtained from BOSTER Biological Technology (Wuhan, China). F4/80+, anti-cleaved caspase-3, TNF- $a$, Smad3, and NOX4 were obtained from Cell Signaling Technology (CST, Danvers, MA, USA). IRDye 800-conjugated secondary antibody was obtained from LI-COR Biosciences (Lincoln, NE, USA). Lipofectamine 2000 was purchased from SciencBio Technology (Invitrogen, Beijing, China).

Mice

C57BL/6 mice used in this study were obtained from Laboratory Animal Center of Anhui province, aged 6-8 weeks, male. Smad3WT and S3KO mice were obtained from the Chinese University of Hong Kong, male, 8 weeks of age, 22-25 g. Animal model of cisplatin-induced acute kidney injury was established by intraperitoneal injection of cisplatin $(20 \mathrm{mg} / \mathrm{kg})$. Mice intraperitoneally injected with saline were used as control. Naringenin $(50 \mathrm{mg} /$ $\mathrm{kg}$ ) was intraperitoneally injected $6 \mathrm{~h}$ before cisplatin treatment and injected daily. All mice were sacrificed under anesthesia 3 days after cisplatin injection. Kidney tissue samples were harvested for PAS staining, immunohistochemistry, Western blot, and real-time PCR, as reported previously, and blood samples were harvested for BUN and Cr detection in accordance with the manufacturer's instructions.

\section{Cell Culture}

Cells were cultured in HyClone ${ }^{\mathrm{TM}}$ DMEM-F12 medium containing $5 \% \mathrm{FBS}$ at $37^{\circ} \mathrm{C}$ and $5 \% \mathrm{CO}_{2}$. Cells were starved for $12 \mathrm{~h}$ with $0.5 \% \mathrm{FBS}$, then treated with cisplatin $(20 \mu \mathrm{M})$ for $24 \mathrm{~h}$ and then harvested and analyzed. A minimum of 3 independent experiments were performed. 


\section{Transfection of Smad3 Small Hairpin RNA}

Smad3 expression was assessed by Western blot and real-time PCR analysis after transfection of mouse tubular epithelial cells (mTEC) cells with mice-derived Smad3 shRNA plasmid obtained from GenePharma Co., Ltd. Briefly, cells were seeded in 6-well plates and transfected with Smad3 shRNA plasmid and control constructs using Lipo2000 transfection reagent (Invitrogen, Carlsbad, CA, USA). Cells were incubated with opti-MEM at $37^{\circ} \mathrm{C}$ and $5 \% \mathrm{CO}_{2}$ for $6 \mathrm{~h}$, then incubated in DMEM containing 5\% FBS. Cells were cultured in DMEM containing 5\% FBS and selected by puromycin to establish Smad3 KD stable cell lines.

\section{Lentivirus-Mediated Smad3 KD in Mice}

Mouse Smad3 shRNA were obtained from GenePharma (Shanghai, China). Lentivirus-mediated Smad3 KD in mice was performed as we previously reported [27], then mice were intraperitoneally injected with either $20 \mathrm{mg} / \mathrm{kg}$ cisplatin or the equal volume of saline or naringenin for further analysis.

\section{Hematoxylin-Eosin Staining}

Kidney tissues were fixed with $4 \%$ paraformaldehyde and then dehydrated, paraffin-embedded, and sliced $(3-5 \mu \mathrm{m})$. The tissue sections were stained with the hematoxylin-eosin (HE) staining reagent performed in Wuhan Servicebio Technology Co., Ltd., for histological analysis and then observed with a microscope (Olympus, Tokyo, Japan). Scoring was performed according to the standard of renal injury: $0=$ normal; $1=<25 \% ; 2=25-50 \% ; 3=50$ $75 \%$; and $4=>75 \%$.

\section{PAS Staining and Immunohistochemical Analysis}

PAS staining was performed with a PAS kit to assess the histological damage, as described previously [27]. Immunohistochemistry was performed on paraffin sections to detect kidney injury by microwave antigen retrieval techniques. Sections were incubated with rabbit anti-KIM1, anti-F4/80+, and anti-TNF- $\alpha$ antibodies overnight at $4^{\circ} \mathrm{C}$. Sections were incubated in secondary antibody and DAB. After immunostaining, the slides were counterstained with hematoxylin and analyzed by Image Analysis System (AxioVision 4; Carl Zeiss, Jena, Germany).

\section{Renal Function Detection}

Blood samples from mice were used to measure $\mathrm{Cr}$ and BUN using Creatinine and BUN Assay Kits according to the manufacturer's instructions.

\section{Western Blot Analysis}

Protein from kidney tissues and cultured cells was extracted with RIPA buffer (Beyotime, Jiangsu, China); BCA kit (Beyotime, Jiangsu, China) was used to measure concentration. Western blot was performed as described previously $[12,27]$. The membrane was incubated with appropriate antibody using rabbit anti-KIM1, anticleaved caspase-3, anti-RIPK1, anti-RIPK3, anti-Smad3, and mouse anti- $\beta$-actin overnight at $4^{\circ} \mathrm{C}$. Membrane was incubated with IRDye 800 -conjugated secondary antibody for $1.5 \mathrm{~h}$ at room temperature. Images were captured with LI-COR/Odyssey infrared image system and then analyzed by Image J software (NIH, Bethesda, MD, USA).

Renal RNA Extraction and Real-Time PCR Examination

Total RNA was obtained from kidney tissues or cultured cells using the RNeasy Isolation Kit according to the manufacturer's instructions (Qiagen, Valencia, CA, USA). Concentration of RNA was measured by a NanoDrop 2000 Spectrophotometer (Thermo scientific, Waltham, MA, USA). Total RNA was reverse-transcribed into cDNA according to the manufacturer's instructions of Bio-Rad kit. Real-time PCR mixture contained $0.3 \mu \mathrm{L}$ upstream and downstream primers for each gene, $4 \mu \mathrm{L}$ Bio-Rad iQ SYBR Green supermix with Opticon2 (Bio-Rad, Hercules, CA, USA), $2.4 \mu \mathrm{L}$ enzyme-free water, and $2 \mu \mathrm{L}$ cDNA solution. The sequences of primers were as follows:mouse KIM1 - forward: $5^{\prime}$-CAGGGAAGCCGCAGAAAA- ${ }^{\prime}$, reverse: $5^{\prime}$-GAGACACGGAAGGCAACCAC-3'; mouse TNF- $\alpha$ - forward: $5^{\prime}$-CATCTTCTCAAAATTCGAGTGACAA-3', reverse: $5^{\prime}$-TGGGAGTAGACAAGGTACAACCC- $3^{\prime}$; mouse MCP-1 - forward: $5^{\prime}$-CTTCTGGGCCTGCTGTTCA-3', reverse: 5'-CCAGCCTACTCATTGGGATCA-3'; mouse NOX4 - forward: $5^{\prime}$-CCCAAGTTCCAAGCTCATTTCC-3', reverse: 5' $^{\prime}$-TGGTGACAGGTTTGTTGCTCCT-3'; mouse IL- $1 \beta$ - forward: $5^{\prime}$-CAACCAACAAGTGATATTCTCCATG-3', reverse: 5'-GATCCACACTCTCCAGCTGCA-3'; mouse IL-6 forward: 5'-GAGGATACCACTCCCAACAGACC-3', reverse : $5^{\prime}$-AAGTGCATCATCGTTGTTCATACA- $3^{\prime}$; mouse IL-8 - forward: $5^{\prime}$-TTCAGAGACAGCAGAGCACA-3', reverse: $5^{\prime}$-GCCAGCTTGGAAGTCATGTT- $3^{\prime}$; mouse $\beta$-actin - forward: $5^{\prime}$-CATTGCTGACAGGATGCAGAA-3' ${ }^{\prime}$, reverse: $5^{\prime}$-ATGGTGCTAGGAGCCAGAGC-3'; mouse Smad3 - forward: 5'-AGGGCTTTGAGGCTGTCTACC-3', reverse: $5^{\prime}$-GTCCACGCTGGCATCTTCTG-3'.

Real-time PCR assay reaction conditions were as follows: denaturation at $95^{\circ} \mathrm{C}$ for $20 \mathrm{~s}$, annealing at $58^{\circ} \mathrm{C}$ for $20 \mathrm{~s}$, elongation at $72^{\circ} \mathrm{C}$ for $20 \mathrm{~s}$, amplification for 40 cycles for each primer. $\beta$-Actin was used to normalize the ratio for the mRNA of other genes.

\section{MTT Assay}

mTEC cells were seeded in 96-well plates and cultured in $\mathrm{Hy}$ Clone $^{\mathrm{TM}}$ DMEM-F12 medium containing $5 \% \mathrm{FBS}$ at $37^{\circ} \mathrm{C}$ and $5 \%$ $\mathrm{CO}_{2}$. Cells were treated by a set of concentrations of naringenin (arranged from 25 to $200 \mu \mathrm{M}$ ) for $12 \mathrm{~h}$, respectively, with or without administration of cisplatin $(20 \mu \mathrm{M})$ for $24 \mathrm{~h}$. Cells were harvested after addition of $5 \mathrm{mg} / \mathrm{mL}$ MTT solution to each well. After $4 \mathrm{~h}$, supernatant was removed and $150 \mu \mathrm{L}$ DMSO was added to dissolve formazan crystals. Optical density was determined in microplate reader (Multiskan MK3, Thermo, Waltham, MA, USA) at $492 \mathrm{~nm}$ wavelength.

\section{Flow Cytometric Analyses}

Flow cytometric analysis was performed to evaluate the percentage of apoptotic cells. The stable mTEC cell lines were treated with $50 \mu \mathrm{M}$ naringenin with or without $20 \mu \mathrm{M}$ cisplatin for 24 h. mTEC cells were digested with trypsin for 2 min and centrifuged at $1,200 \mathrm{rpm}$ for $5 \mathrm{~min}$ at $4^{\circ} \mathrm{C}$. According to the manufacturer's instructions, the density of cells used was $10^{6}$ cells $/ \mathrm{mL}$ after addition of $400 \mu \mathrm{L}$ Annexin V-binding fluid. Cells were restained with $10 \mu \mathrm{L}$ PI for 5 min and lightly placed at $4^{\circ} \mathrm{C}$ in dark before being immediately measured with a laser 8-color flow cytometer (FACS Verse, BD, USA) and quantified using Flow Jo 7.6 software [27].

\section{ELISA}

The levels of interleukin (IL)- $1 \beta$ and monocyte chemotactic protein-1 (MCP-1) were measured by ELISA (R\&D Systems) according to the manufacturer's instructions. 
PAS

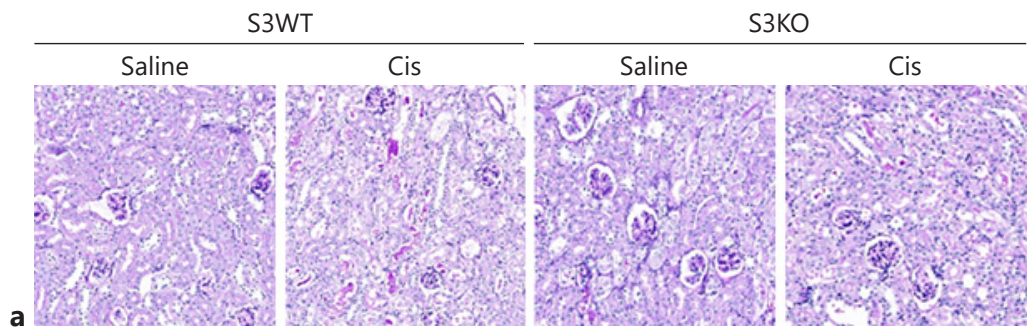

HE staining

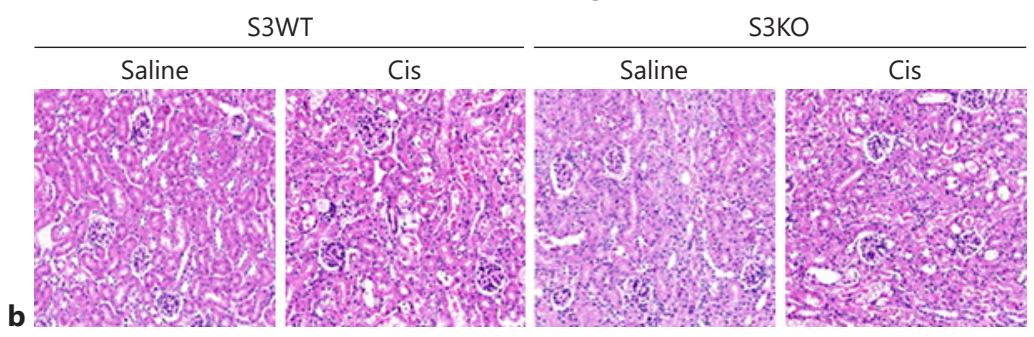

Western blot

KIM1 protein

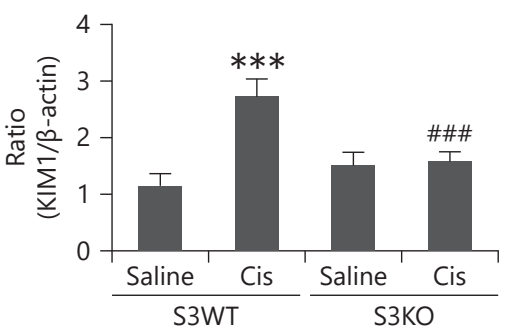

Cleaved caspase-3 protein

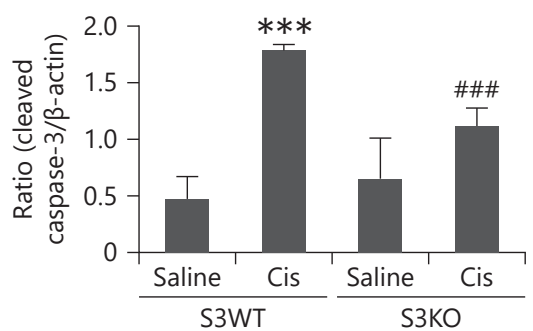

Immunohistochemistry

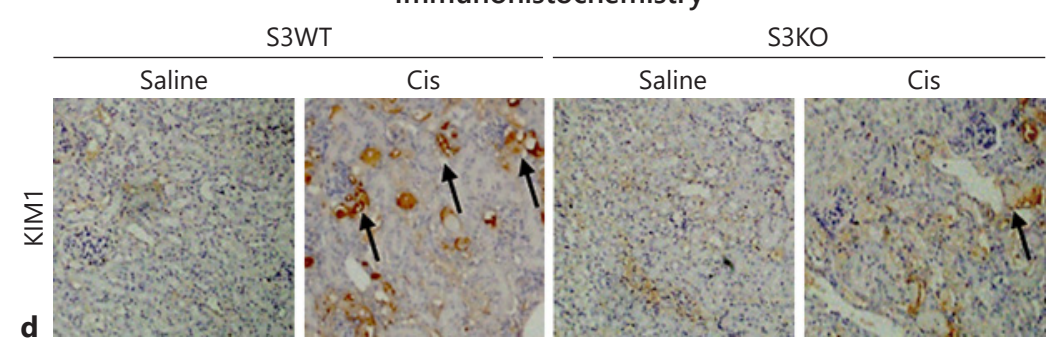

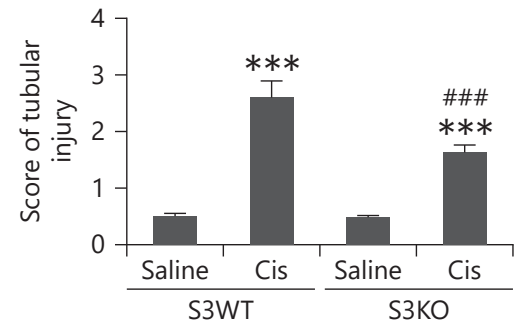

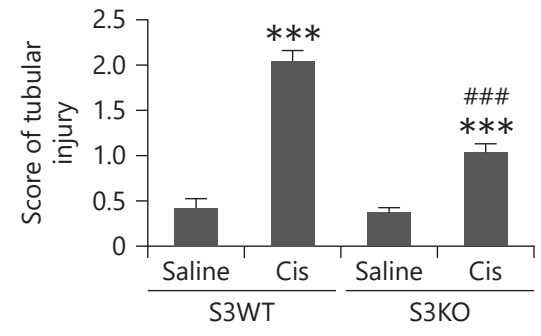

RIPK1 protein

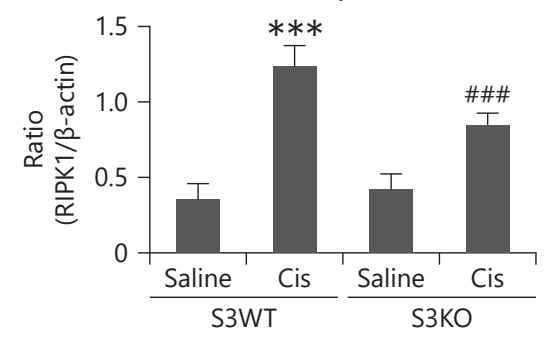

RIPK3 protein

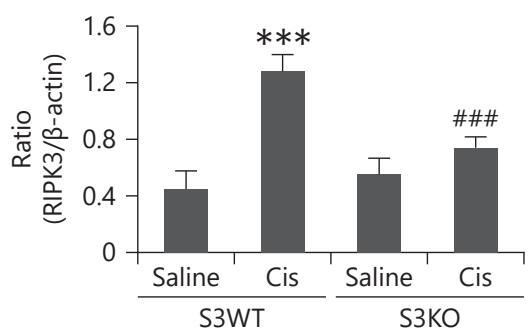

Quantification of KIM1

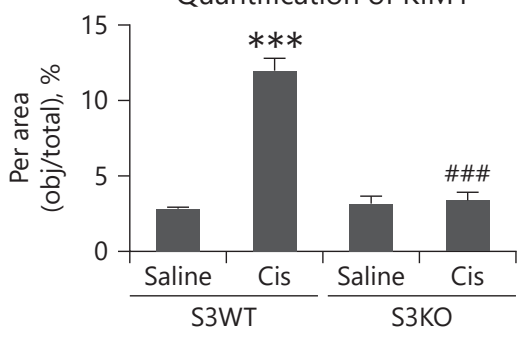

(For legend see next pages.)

Smad3-Targeted Therapy Protects against AKI
Kidney Dis 2021;7:372-390

DOI: $10.1159 / 000512986$ 


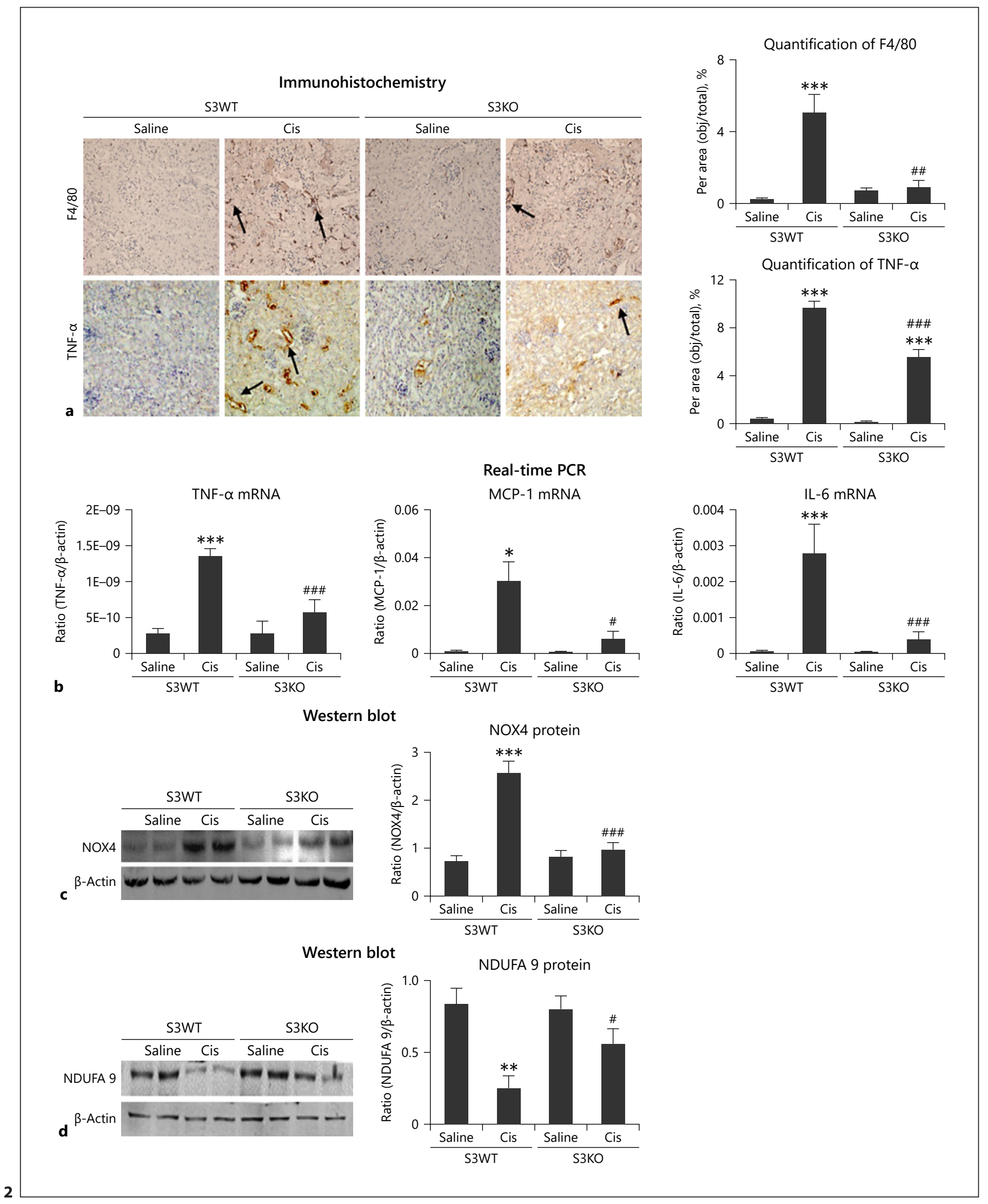

(For legend see next page.) 


\section{DHE Staining}

The oxidation of DHE, fluorescent red and ethidium bound to DNA, was used to estimate intracellular reactive oxygen species (ROS) levels according to the manufacturer's instructions. Cells were incubated with $5 \mathrm{~mm}$ freshly prepared DHE solution (Beyotime, Jiangsu, China) for $30 \mathrm{~min}$ at $37^{\circ} \mathrm{C}$ and then measured under fluorescence microscopy.

\section{DCF Assay}

DCF, the oxidation product of 2,7-dichlorodihydrofluorescein diacetate, is a marker of cellular oxidation. According to the manufacturer's instructions, the ROS level was measured by fluorescence microscopy with excitation of $488 \mathrm{~nm}$ and emission of 525 $\mathrm{nm}[1]$.

\section{Immunofluorescence}

mTEC cells were cultured in 8-chamber glass slides in the presence or absence of cisplatin $(20 \mu \mathrm{M})$ for $24 \mathrm{~h}$ after incubation with naringenin overnight. Cells were then fixed in $4 \%$ paraformaldehyde and incubated with the antibodies detecting mouse antiSmad 3 and rabbit anti-NOX 4 at $4{ }^{\circ} \mathrm{C}$ overnight, followed by $1 \mathrm{~h}$ of incubation with goat anti-rabbit and goat anti-mouse IgG rhodamine (Bioss Biotechnology, Beijing, China). Cells were then counterstained with DAPI and analyzed under fluorescence microscope (Zeissspot; Carl Zeiss Micro Imaging GmbH, Gottingen, Germany), as previously described [28].

\section{Co-Immunoprecipitation}

mTEC cells were harvested with 1\% NP-40 followed by centrifugation at $3,000 \mathrm{rpm}$ for $5 \mathrm{~min}$. Protein was incubated with NOX 4 antibody for $2 \mathrm{~h}$ at $4^{\circ} \mathrm{C}$. Then, capture the immunocomplex by adding $100 \mu \mathrm{L}$ of washed Protein A agarose bead slurry (EMD Millipore Corporation, 28820 Single Oak Drive, Temecula, CA, USA). The tagged protein was incubated with the bead for $12 \mathrm{~h}$ at $37^{\circ} \mathrm{C}$ to get the protein-bead complex. Protein-bead complex was washed with $1 \% \mathrm{NP}-40$ for 3 cycles. Samples were finally measured by Western blot with Smad3 antibody.

\section{Chromatin Immunoprecipitation Assay}

Chromatin immunoprecipitation assay was performed using the SimpleChIP Enzymatic Chromatin IP Kit (Magnetic Beads) \#9003 (Cell Signaling Technology, Beverly, MA, USA) according to the manufacturer's instructions. Cells were stimulated with cisplatin for $24 \mathrm{~h}$ and fixed with $1 \%$ formaldehyde in DMEM for 10 min. Glycine was added to each dish and set for $5 \mathrm{~min}$ prior to washing in cold PBS 2 times. Cells were collected, resuspended, and sonicated to generate $200-800$ bp DNA fragments. Subsequently, the following antibodies were used to immunoprecipitate cross-linked protein-DNA complexes: rabbit anti-Smad3 and normal rabbit IgG. The immunoprecipitated DNA was purified for PCR analysis with primers specific for the putative binding sites within the promoter of NOX4.

\section{Statistical Analysis}

The data acquired from this study are presented as the mean \pm SEM from 3 to 4 independent in vitro experiments or 6-8 mice. Statistical analyses were performed using 2-tailed unpaired $t$ test or 1-way ANOVA, followed by Newman-Keuls post hoc test (Prism 5.0; GraphPad Software, San Diego, CA, USA).

\section{Results}

\section{KO of Smad3 Attenuated Cisplatin-Induced Renal} Injury, Programmed Cell Death, Inflammation, and

\section{Oxidative Stress in vivo}

PAS and HE staining showed Smad3 KO attenuated renal tubule enlargement and cell damage in cisplatin nephropathy (Fig. 1a, b). We then determined the role of Smad3 in cisplatin-induced renal injury and programmed cell death. Western blot revealed decreased levels of KIM1, RIPK1, RIPK3, and cleaved caspase-3 in Smad3 $\mathrm{KO}$ mice (Fig. 1c). Consistently, immunohistochemistry revealed that KIM1 was significantly lower in Smad3 KO mice than in S3WT mice (Fig. 1d). Furthermore, Smad3 $\mathrm{KO}$ mice also showed reduced $\mathrm{F} 4 / 80+$ macrophage infiltration and TNF- $\alpha$ signals (Fig. 2a). This result was further confirmed by real-time PCR, which showed reduced mRNA levels of inflammatory indicators including
Fig. 1. Smad3 KO prevented cisplatin-induced renal injury and programmed cell death in vivo.a PAS staining. Smad3 KO reduced renal injury in cisplatin nephropathy. b HE staining. c Western Blot analysis of KIM1, RIPK1, RIPK3, and cleaved caspase-3. d Immunohistochemistry and quantitative data of KIM1. Smad3 KO reduced the percentage of KIM1-positive cells in injured kidney, as the arrows shown in pictures. Data are mean \pm SEM for 6-8 mice. ${ }^{* * *} p<0.001$ versus normal; \#\#\# $p<0.001$ versus Smad3 WT + cisplatin group. Magnification, $\times 20$. KO, knockout; PAS, periodic acid-Schiff; HE, hematoxylin-eosin; WT, wild type.
Fig. 2. Smad3 KO attenuated cisplatin-induced renal inflammation and NOX4-dependent oxidative stress in vivo. a Immunohistochemistry of F4/80+ and TNF- $\alpha$. Smad3 KO reduced inflammatory response, including recruitment of macrophages and secretion of TNF- $\alpha$, as the arrows shown in pictures. b Realtime PCR for inflammatory indicators in mice. Smad3 KO reduced
mRNA levels of TNF- $\alpha$, IL- 6 , and MCP-1 compared with those in S3WT mice. c Western blot analysis of NOX4. d Western blot analysis of NDUFA 9. Data represent mean \pm SEM for $6-8$ mice. ${ }^{*} p<$ $0.05,{ }^{* *} p<0.01,{ }^{* * *} p<0.001$ versus normal; $\# p<0.05, \# \# p<0.01$, $\# \#$ \# $<0.001$ versus S3FF + cisplatin group. Magnification: $\times 20$. $\mathrm{KO}$, knockout; WT, wild type. 
Western blot (mTEC)
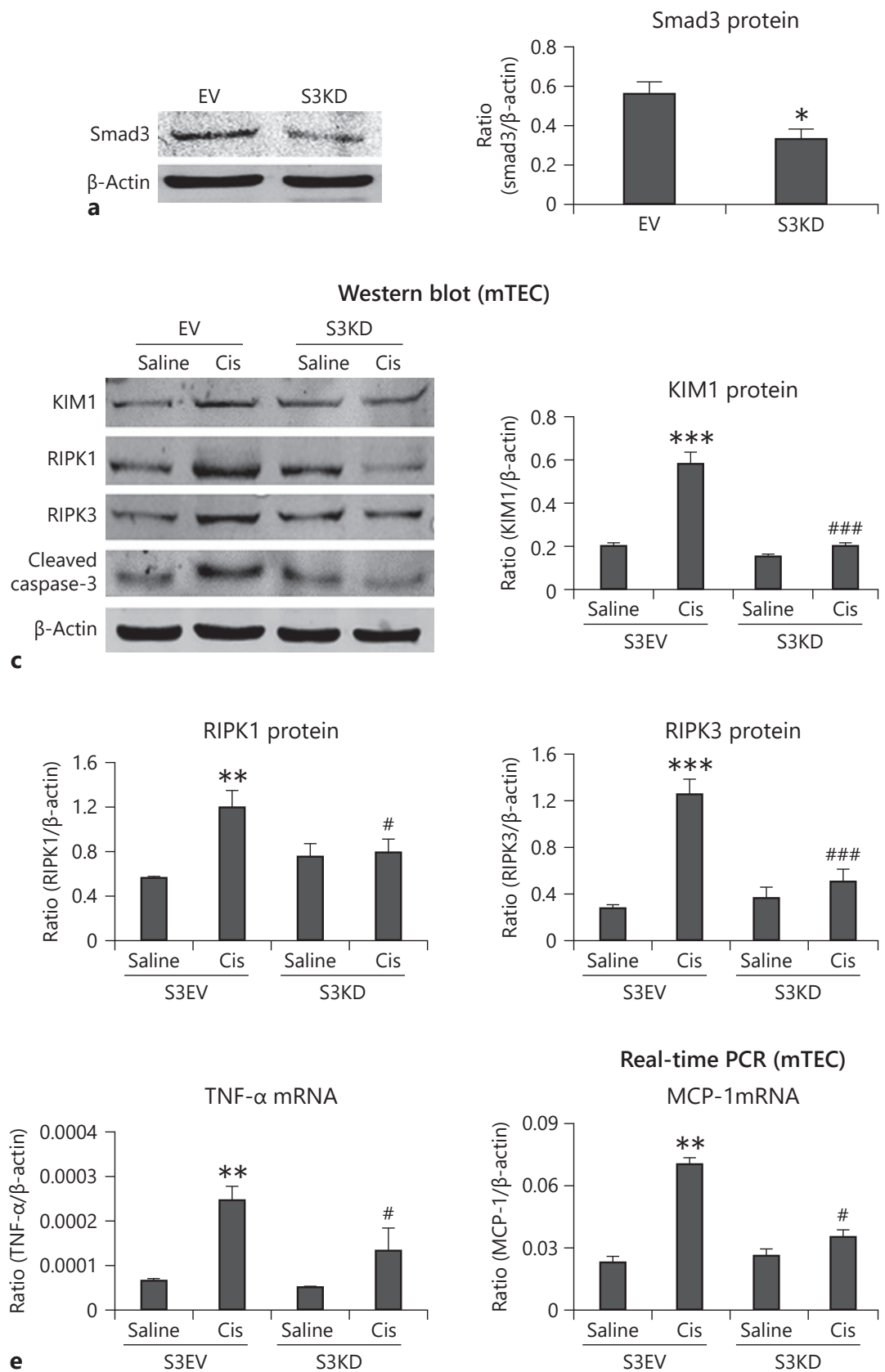

Real-time PCR (mTEC)

KIM1 mRNA

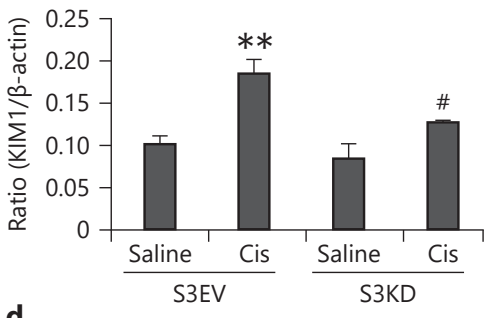

Cleaved caspase-3 protein

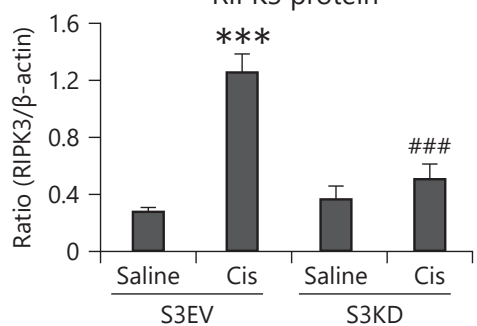

Real-time PCR (mTEC)
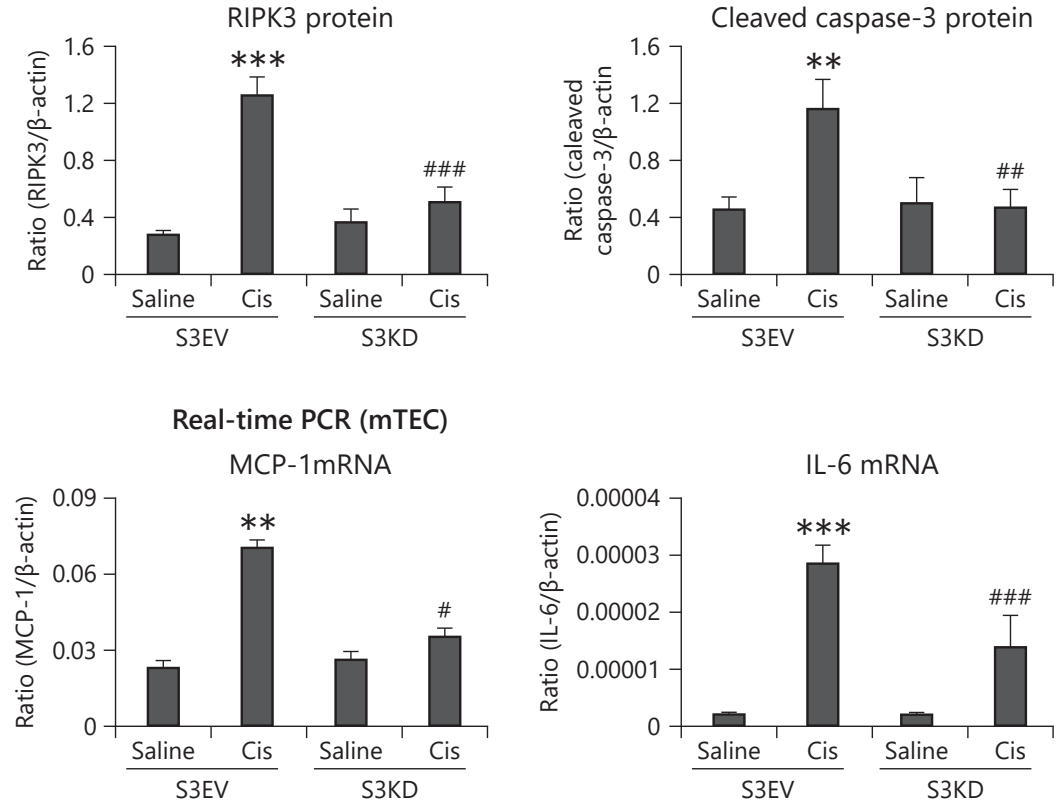

Real-time PCR (mTEC) Smad3 mRNA

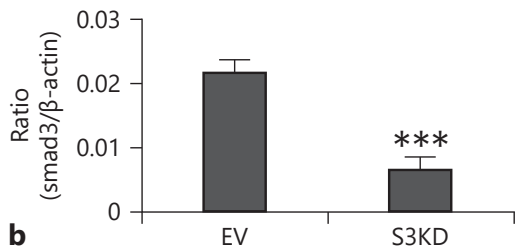

Fig. 3. Smad3 KD attenuated cell injury, programmed cell death, and inflammation in cisplatin-treated tubular epithelial cells. a, b Western blot and real-time PCR of Smad3. Smad3 KD was confirmed in mTEC. c Western blot and quantitative data of KIM1, RIPK1, RIPK3, and cleaved caspase-3. d Real-time PCR of KIM1. e Real-time PCR shows that Smad3 KD reduced mRNA levels of
TNF- $\alpha$, IL-6, and MCP-1. Data represent the mean \pm SEM for 3-4 independent experiments. ${ }^{*} p<0.05,{ }^{* *} p<0.01,{ }^{* * *} p<0.001$ versus control; $\# p<0.01, \# \# p<0.01$, \#\#\#p<0.001 versus Smad3 vector control + cisplatin. EV, empty vector; KD, knockdown; mTEC, mouse tubular epithelial cells. 


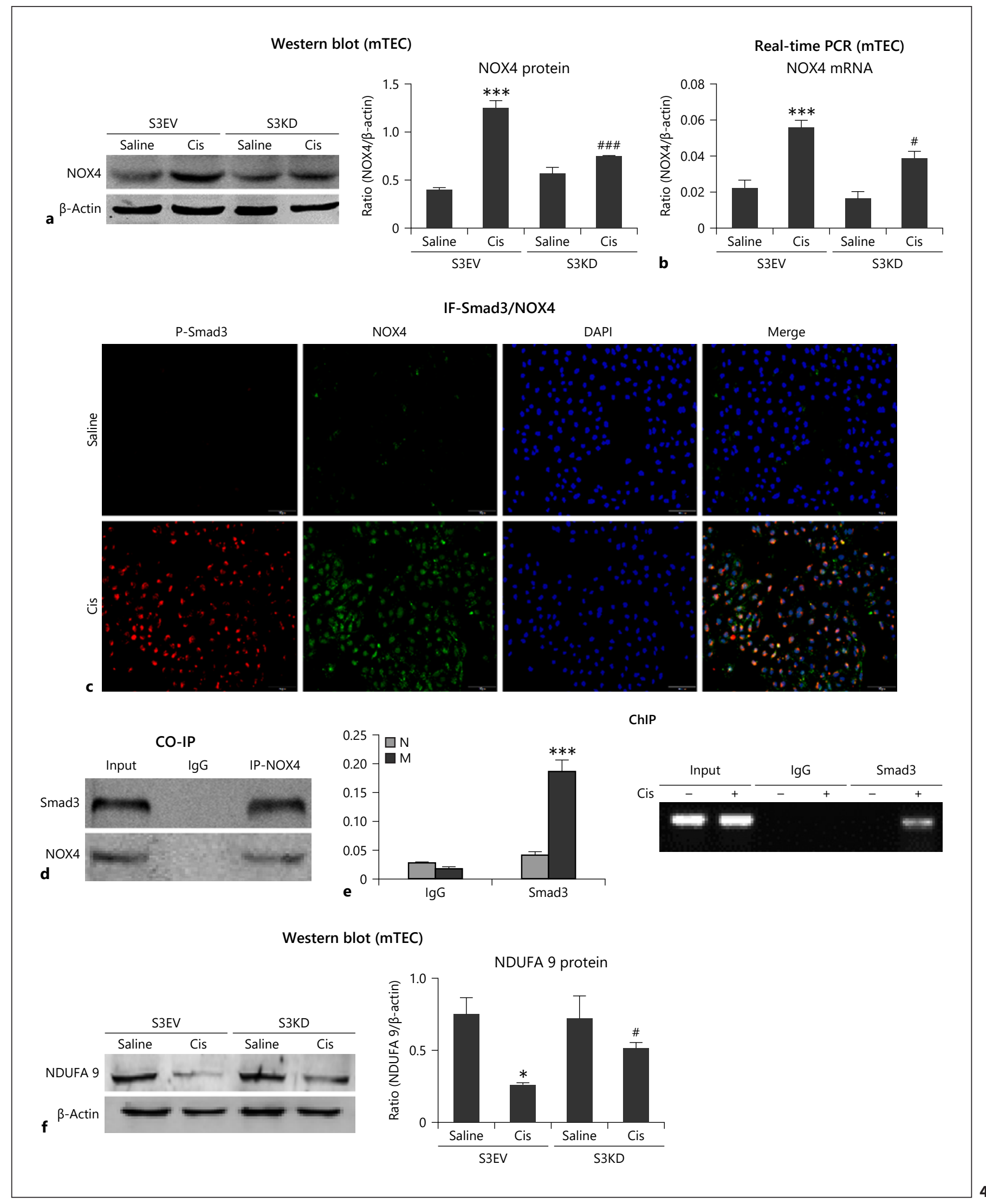

(For legend see next pages.)

Smad3-Targeted Therapy Protects against AKI
Kidney Dis 2021;7:372-390

DOI: $10.1159 / 000512986$ 


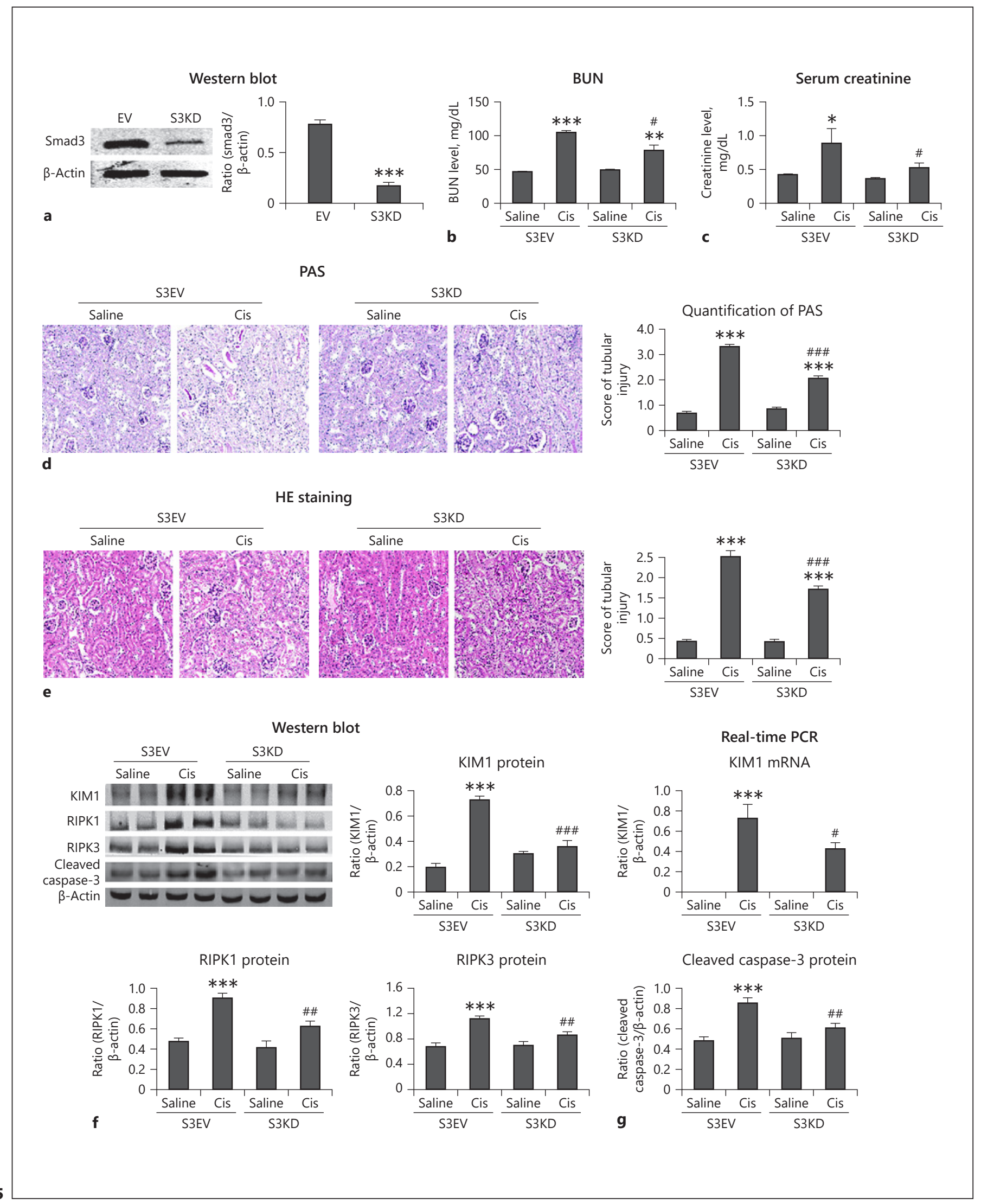

(For legend see next page.) 
TNF- $\alpha$, IL-6, and chemokine MCP-1 (Fig. 2b). Moreover, Western blot results showed significant reduction in NOX4 levels in cisplatin-stimulated Smad3 $\mathrm{KO}$ mice compared with those in S3WT mice (Fig. 2c) and recovered protein levels were found for complex I subunits, such as NDUFA 9 (Fig. 2d). These findings show that Smad3 promotes cisplatin-induced tubular injury, inflammation, programmed cell death, and ROS in vivo.

\section{Cisplatin-Induced Cell Injury, Programmed Cell}

Death, Inflammation, and Oxidative Stress Were

Attenuated in Smad3 KD TEC

We next determined the function of Smad3 in TECs. Western blot and real-time PCR revealed significant decrease in Smad3 protein and mRNA levels in Smad3 KD TEC (Fig. 3a, b). Consistently, Western blot revealed significant reduction in KIM1, RIPK1, RIPK3, and cleaved caspase-3 levels in cisplatin-stimulated Smad3 KD cells compared with those in cells transfected with empty vector (Fig. 3c). The mRNA levels of KIM1, TNF- $\alpha$, IL-6, and MCP-1 were significantly decreased in Smad3 KD cells (Fig. 3d, e). Additionally, Smad3 KD reduced cisplatininduced NOX4-dependent oxidative stress in vitro (Fig. 4a, b). We next examined the mechanisms of Smad3 and NOX4. Co-immunoprecipitation and immunofluorescence analyses confirmed the interaction between Smad3 and NOX4 (Fig. 4c, d). Interestingly, Smad3 bound to the promoter region of NOX4 to induce the production of NOX4 (Fig. 4e), indicating that Smad3 regulates NOX4 expression and function at 2 different levels and that this interaction with NOX4 could be the major mechanism through which cisplatin induces oxidative stress. Moreover, Smad3 KD recovered protein levels of NDUFA 9 (Fig. 4f).

Fig. 4. Cisplatin-induced oxidative stress was relieved in Smad3 KD cells. a, b Western blot and real-time PCR of NOX4. c Immunofluorescence of Smad3 and NOX4. d Co-immunoprecipitation. Smad3 interacted with NOX4 to regulate its function. e ChIP assay. Smad3 bound the promoter region of NOX4. $\mathbf{f}$ Western blot

Fig. 5. Lentivirus-mediated Smad3 KD effectively reduced cisplatin-induced injury and programmed cell death in vivo. a Western blot of NOX4. Smad3 KD was confirmed in vivo. b, c BUN and serum $\mathrm{Cr}$ analyses. Smad3 silencing reduced cisplatin-induced renal injury. d PAS staining. Smad3 KD reduced renal injury in cisplatin nephropathy. e HE staining. $\mathbf{f}$ Western blot and quantitative data. Protein levels of KIM1, RIPK1, RIPK3, and cleaved caspase-3
Lentivirus-Mediated Smad3 KD Effectively Reduced Cisplatin-Induced Renal Injury, Inflammation, Programmed Cell Death, and Oxidative Stress in vivo

Western Blot confirmed Smad3 KD in mice (Fig. 5a). Further, Smad3 silencing reduced cisplatin-induced renal injury, and this finding was confirmed by detecting the serum $\mathrm{Cr}$ and BUN (Fig. 5b, c). PAS staining and HE staining showed Smad3 KD attenuated kidney damage in cisplatin nephropathy (Fig. 5d, e). Furthermore, protein levels of KIM1, RIPK1, RIPK3, and cleaved caspase-3 were significantly reduced in cisplatin-injured kidneys of Smad3 KD mice compared with those in empty vector control (Fig. 5f, g). Immunohistochemistry revealed that $\mathrm{Smad} 3 \mathrm{KD}$ reduced the expression of KIM1, TNF- $\alpha$, and F4/80+ macrophage infiltration (Fig. 6a). Additionally, real-time PCR revealed decreased mRNA levels of TNF- $\alpha$, IL- 6 , and MCP-1 in Smad3 KD mice (Fig. 6b). Western blot and real-time PCR further showed that NOX4 was significantly reduced in cisplatin-injured kidneys of Smad3 KD mice (Fig. 6c, d). Western blot showed that the protein level of NDUFA 9 was recovered in Smad3 KD mice (Fig. 6e). These findings suggest that lentivirus-mediated Smad3 silencing can effectively attenuate cisplatin-induced renal injury, inflammation, programmed cell death, ROS production, and oxidative respiratory chain of complex I subunits in vivo.

\section{Inhibition of Smad3 Using Naringenin Attenuated} Cisplatin-Induced Kidney Injury, Programmed Cell

Death, Inflammation, and Oxidative Stress in vitro

We found that naringenin had low cytotoxicity, $<100$ $\mu \mathrm{M}$, and it exerted protective effects in cisplatin-treated mTEC (Fig. 7a, b). Western blot showed naringenin sig-

analysis of NDUFA 9. Data represent the mean \pm SEM for 3-4 independent experiments. ${ }^{* * *} p<0.001$ versus control; $\# p<0.01$, $\# \# \#<0.001$ versus Smad3 vector control + cisplatin. EV, empty vector; KD, knockdown; mTEC, mouse tubular epithelial cells.

were reduced in Smad3 KD mice. g Real-time PCR of KIM1. Smad3 KD significantly reduced the mRNA level of KIM1 in vivo. Data are mean \pm SEM for $6-8$ mice. ${ }^{*} p<0.05,{ }^{* *} p<0.01,{ }^{* * *} p<$ 0.001 versus control; $\# p<0.01$, \#\#p $<0.01$, \#\#\#p<0.001 versus $S$ mad3 vector control + cisplatin. Magnification, $\times 20$. PAS, periodic acid-Schiff; EV, empty vector; KD, knockdown. 


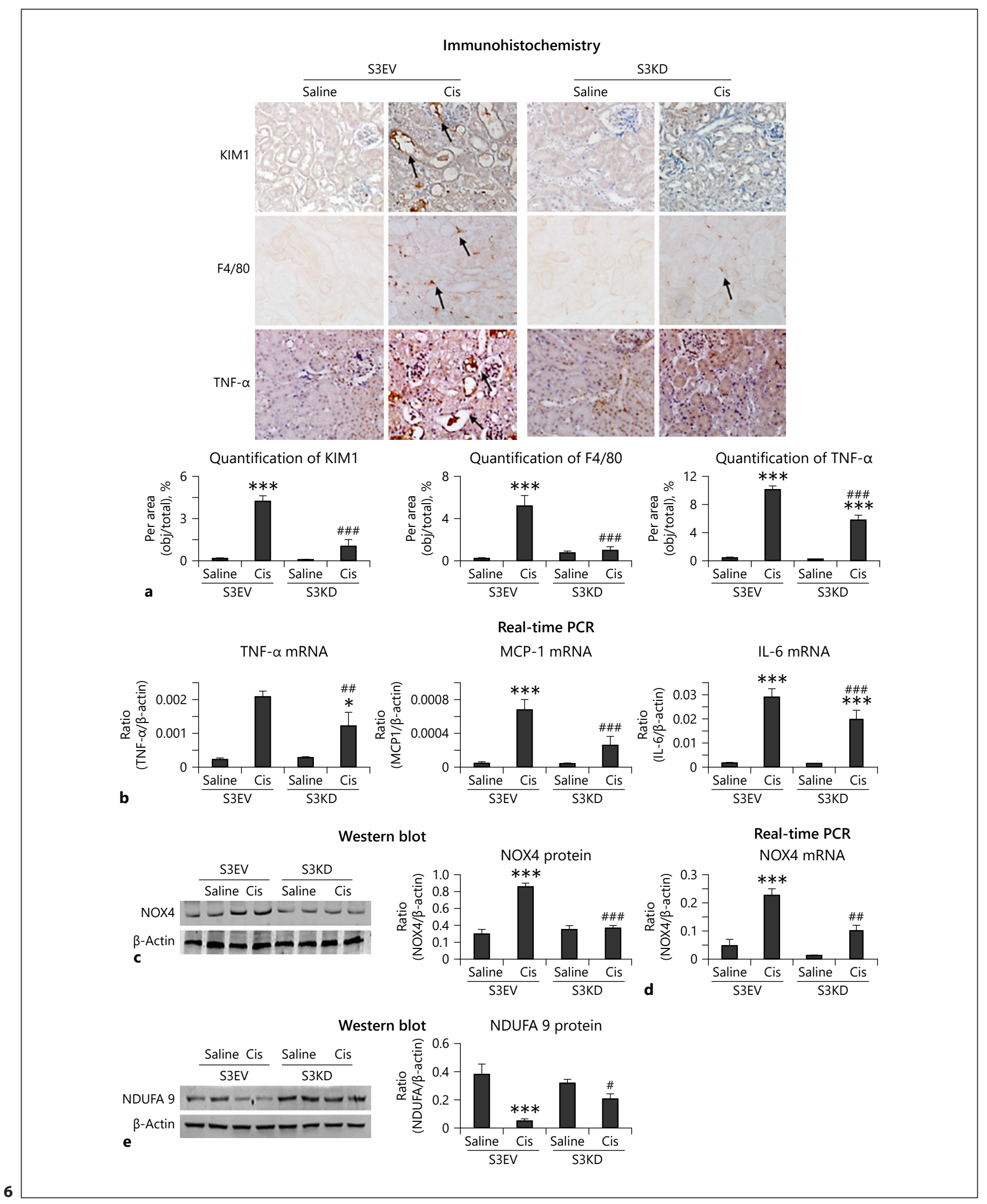

(For legend see next pages.) 

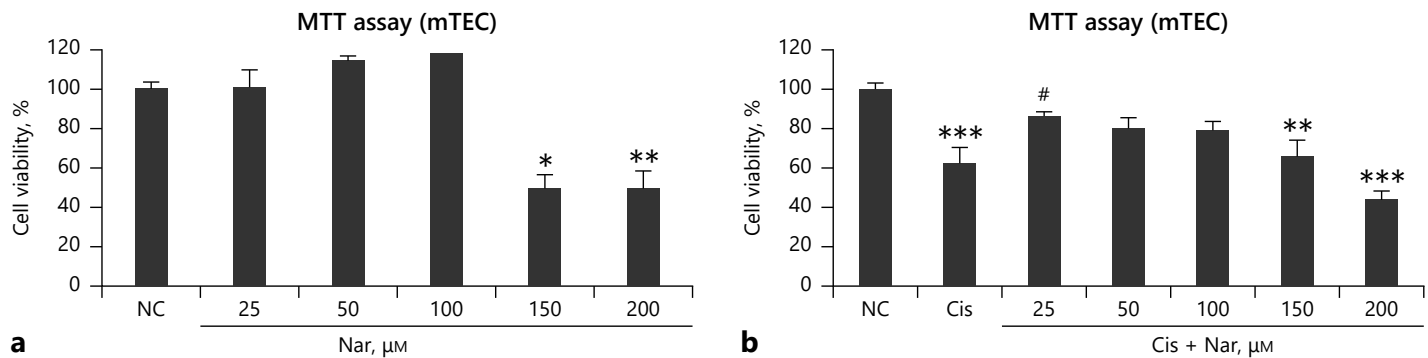

Western blot (mTEC)
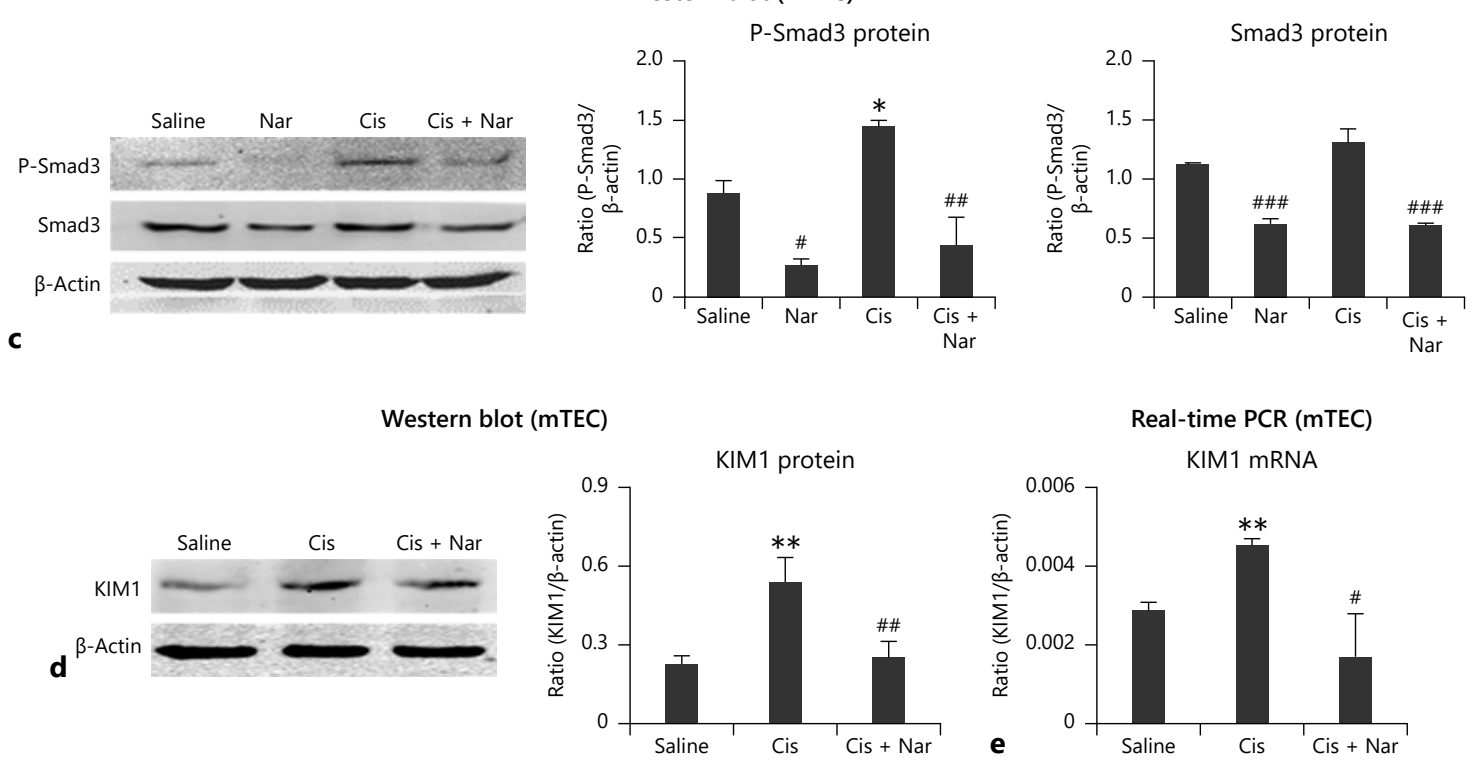

Flow cytometry (mTEC)
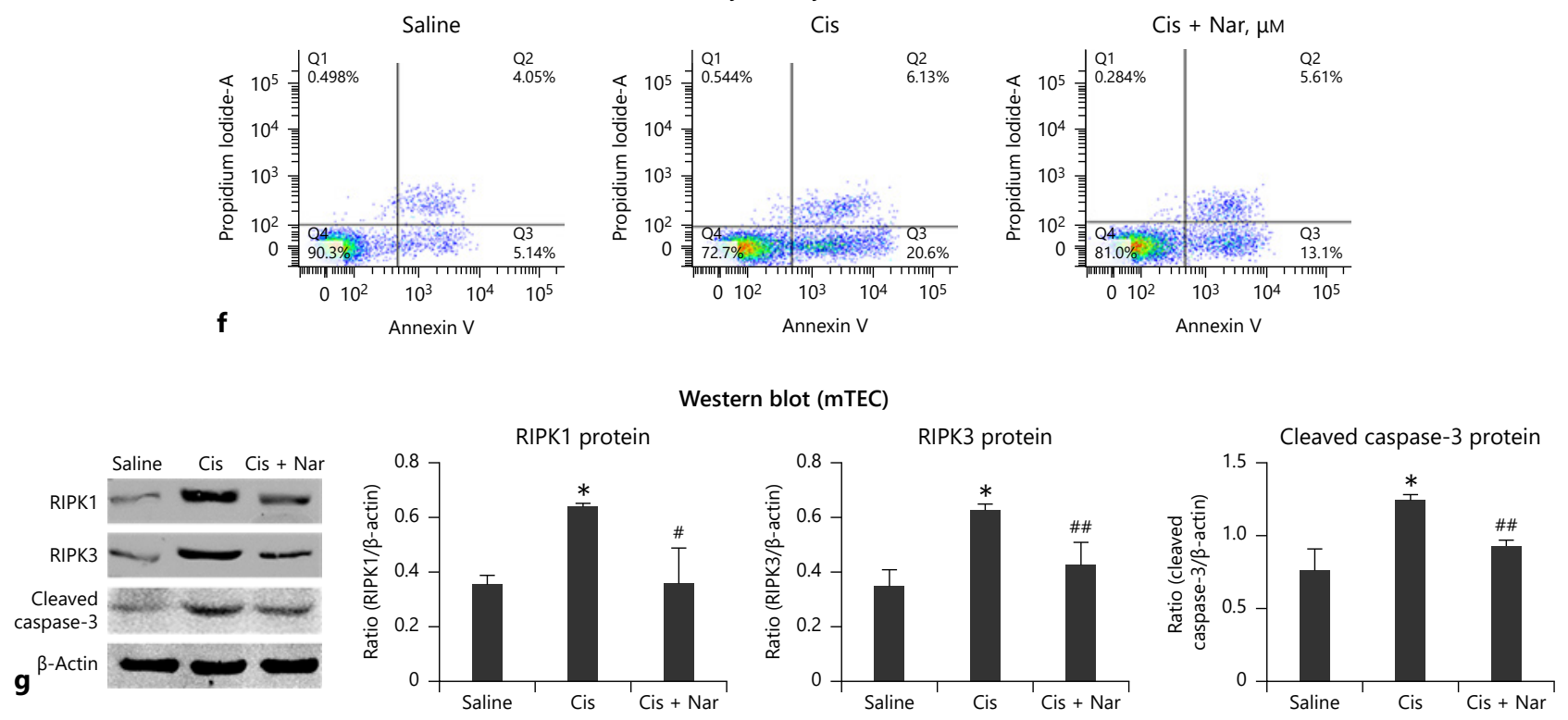

(For legend see next page.) 
nificantly reduced P-Smad3 and Smad3 protein levels (Fig. 7c). Western blot and real-time PCR showed significant reduction in KIM1 protein and mRNA levels in cells treated with cisplatin (Fig. 7d, e). In addition, flow cytometry and Western blot for RIPK1, RIPK3, and cleaved caspase- 3 revealed that naringenin effectively attenuated cisplatin-induced programmed cell death in vitro (Fig. 7f, g). Furthermore, real-time PCR and ELISA revealed that inflammation was alleviated in the naringenin treatment group (Fig. 8a, b). In addition, Smad3 inhibition by treatment with naringenin reduced cisplatin-induced NOX4-dependent oxidative stressin vitro (Fig. 8c-f).

Smad3-Targeted Therapy Using Naringenin Attenuated Cisplatin-Induced Kidney Injury,

Programmed Cell Death, Inflammation, and

Oxidative Stress in vivo

Naringenin treatment reduced kidney damage and recovered the renal function in vivo (Fig. 9a-d). Western blot and real-time PCR revealed significant reduction in KIM1 levels in naringenin-treated mice (Fig. 9e, f). In addition, the protein levels of RIPK1, RIPK3, and cleaved caspase- 3 were significantly reduced by naringenin (Fig. 9g). Real-time PCR revealed that naringenin protected against cisplatin-induced inflammation (Fig. 10a), and this result was confirmed by the immunohistochemistry of KIM1, F4/80+, and TNF-a (Fig. 10b). Additionally, Western blot and real-time PCR showed that naringenin significantly reduced the protein and mRNA levels of NOX4 in vivo (Fig. 10c, d).

Fig. 6. Lentivirus-mediated $\mathrm{Smad} 3 \mathrm{KD}$ reduced cisplatin-induced renal inflammation and NOX4-dependent oxidative stress in vivo. a Immunohistochemistry for detection of KIM1, TNF- $\alpha$, and F4/80+ macrophages. Smad3 KD decreased infiltration of F4/80+ macrophages and protein levels of KIM1 and TNF- $\alpha$ in cisplatin nephropathy, as the arrows shown in figures. b Real-time PCR. Smad3 KD reduced the mRNA levels of TNF- $\alpha$, IL-6, and MCP-1.

Fig. 7. Treatment with naringenin reduced cisplatin-induced injury and programmed cell death in mTEC. a, b MTT assay. Naringenin had low cytotoxicity and exerted protective effects on cisplatin-treated mTEC. c Western blot of P-Smad3 and Smad3. d, e Western blot and real-time PCR of KIM1. Naringenin treatment significantly reduced protein and mRNA levels of KIM1 in cisplatin-treated mTEC. f Flow cytometry of PI/Annexin V. g Western

\section{Discussion}

The current study found that $\mathrm{Smad} 3 \mathrm{KO}$ attenuated the loss of renal function, cell apoptosis, necroptosis, oxidative stress, and inflammation in cisplatin nephropathy. This finding was consistent with our in vitro study showing that Smad3 KD prevented oxidative stress, programmed cell death, and inflammatory response in cisplatin-treated TECs. Furthermore, Smad3targeted therapy using 2 protocols was tested in vivo, and the results demonstrated that lentivirus-mediated Smad3 KD could protect against renal injury caused by cisplatin injection, whereas treatment with naringenin, a Smad3 inhibitor and a monomer used in traditional Chinese medicine, protects against AKI. Mechanistically, Smad3 bound the promoter region of NOX4 to induce the production of NOX4, while it also interacted with NOX4 to induce NOX4-dependent ROS production. This might be one of the major mechanisms mediating cisplatin-induced programmed cell death and inflammation.

First, in this study, we explored the mechanisms of Smad3 in renal injury, programmed cell death, and inflammation. Necroptosis is regulated by the death signaling pathway and is a recently identified cell death mechanism that has a key role in AKI. Necroptotic cells release components such as high mobility group protein to induce severe necroinflammation [29]. Emerging evidence shows that RIPK1, RIPK3, and MLKL are central regulators in the necroptotic pathway [30-32]. Our team recently found that hsa-miR-500a-3P directly targets the

c, d Western blot and real-time PCR of NOX4. NOX4 was significantly reduced in cisplatin-injured kidneys of Smad3 KD mice. e Western blot analysis of NDUFA 9. Data are mean \pm SEM for 6-8

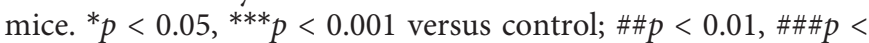
0.001 versus $S m a d 3$ vector control + cisplatin. Magnification, $\times 20$. $\mathrm{EV}$, empty vector; KD, knockdown.

blot analysis and quantitative data of RIPK1, RIPK3, and cleaved caspase-3. Naringenin inhibited cisplatin-induced necrosis and apoptosis in mTEC. Data represent the mean \pm SEM for 3-4 independent experimentsin vitro. ${ }^{* *} p<0.05,{ }^{* *} p<0.01,{ }^{* * *} p<0.001$ versus normal; $\# p<0.05$, \#\#p<0.01 compared to cisplatin-treated group. mTEC, mouse tubular epithelial cells; Nar, naringenin. 

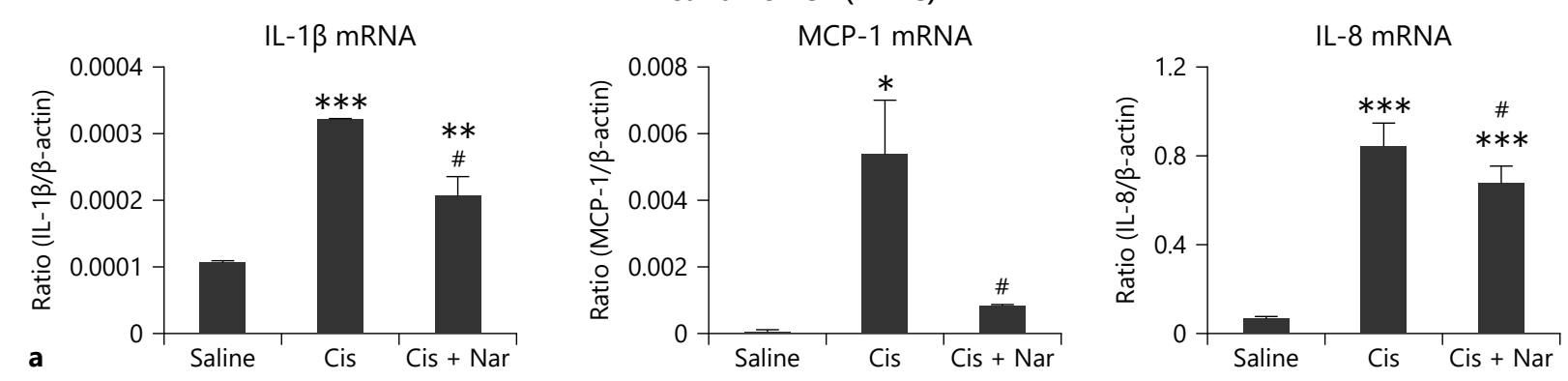

\section{ELISA (mTEC)}
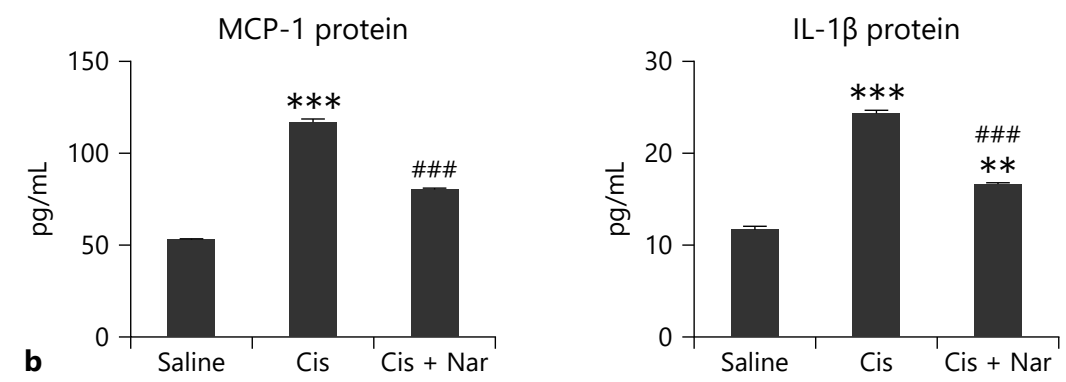

Western blot (mTEC)
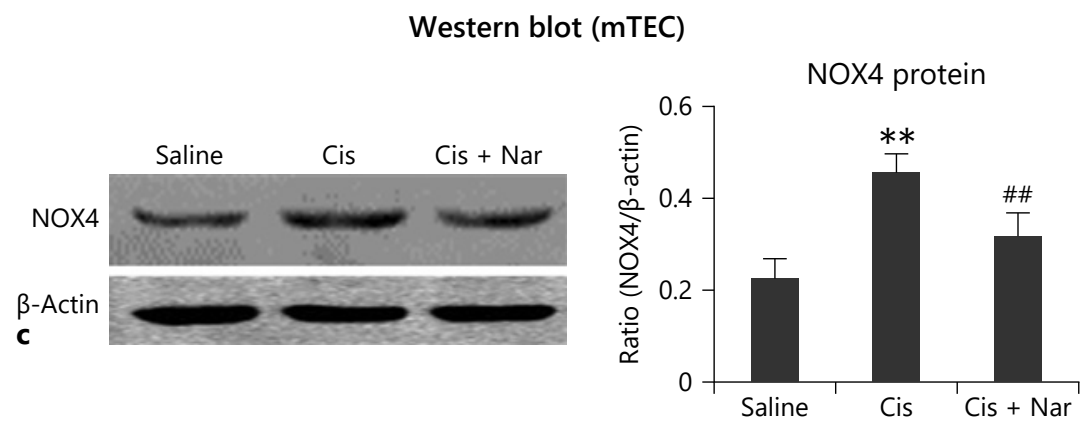

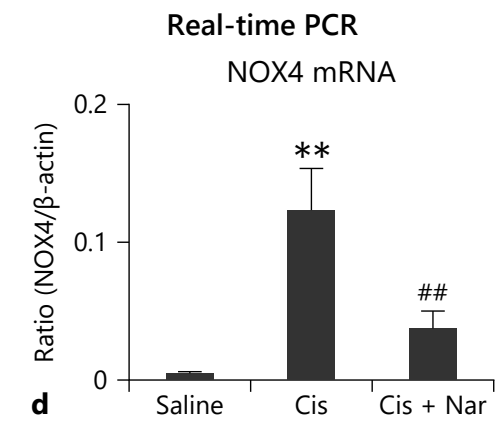

\section{DHE staining}

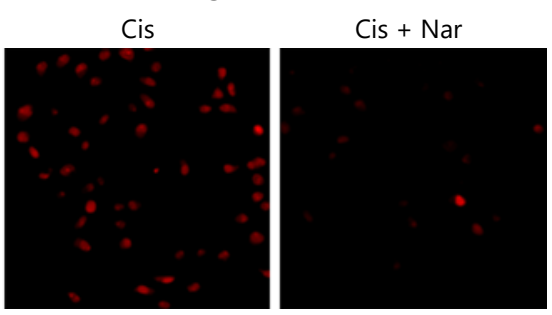

Fig. 8. Treatment with naringenin reduced cisplatin-induced inflammatory response and oxidative stress in mTEC. $\mathbf{a}, \mathbf{b}$ Real-time PCR and ELISA of mTEC cells. Inflammation was alleviated in the naringenin treatment group. c, d Western blot and real-time PCR of NOX4. e, $\mathbf{f}$ DCF assay of reactive oxygen species and DHE staining in cisplatin-treated mTEC. Smad3-targeted therapy using nar- ingenin reduced cisplatin-induced NOX4-dependent oxidative stressin vitro. Data represent the mean \pm SEM for 3-4 independent experiments in vitro. ${ }^{* *} p<0.05,{ }^{* *} p<0.01,{ }^{* * *} p<0.001$ versus normal; $\# p<0.05$, \#\#p<0.01 compared to cisplatin-treated group. mTEC, mouse tubular epithelial cells; Nar, naringenin. 

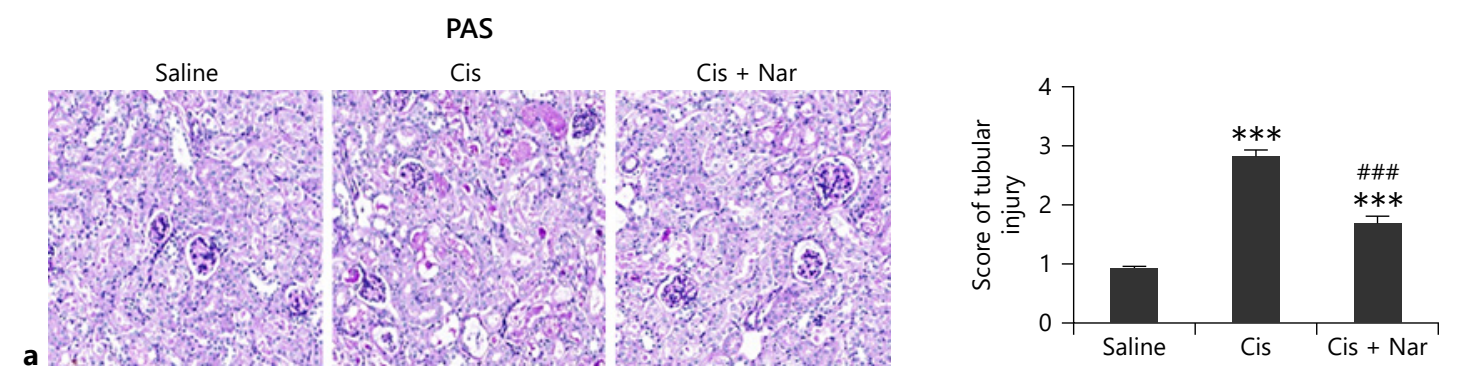

HE staining
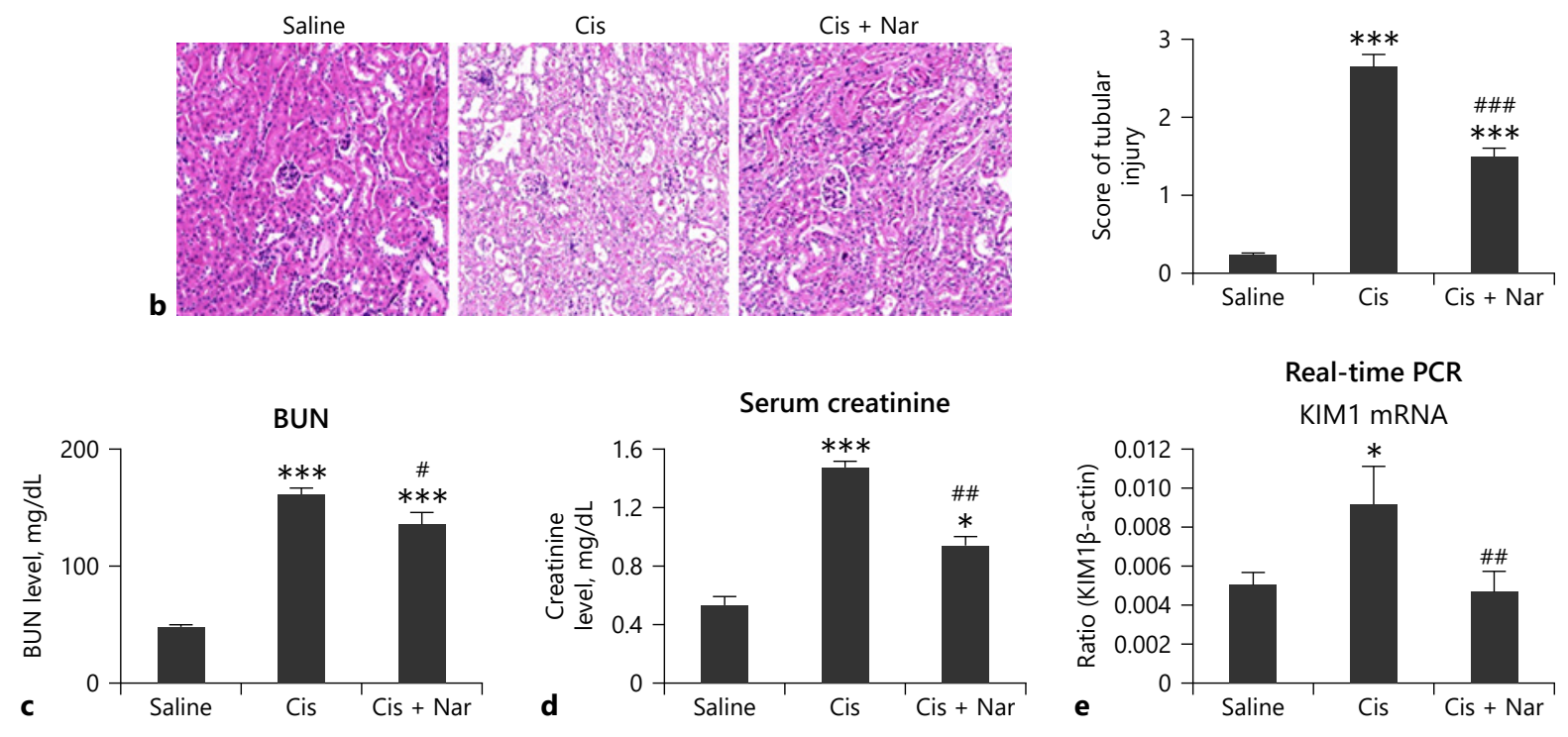

Western blot
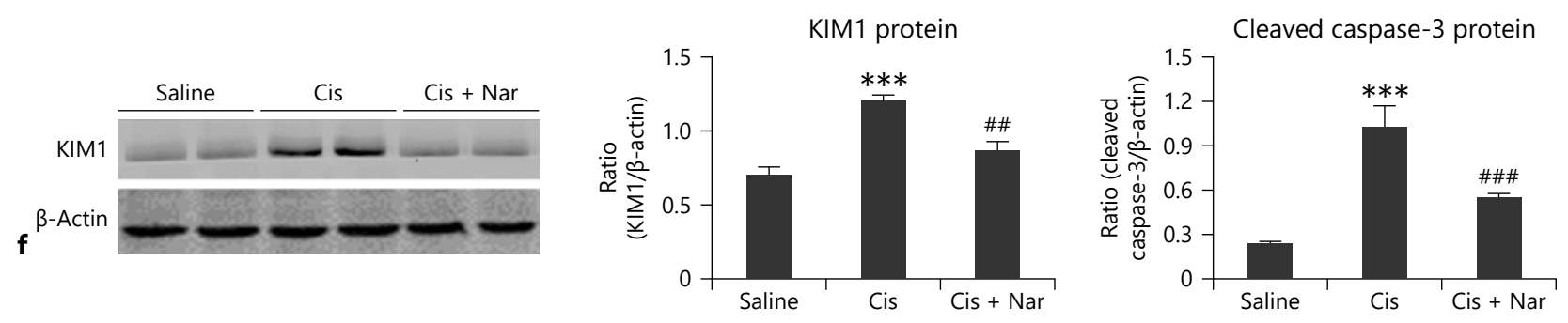

Western blot
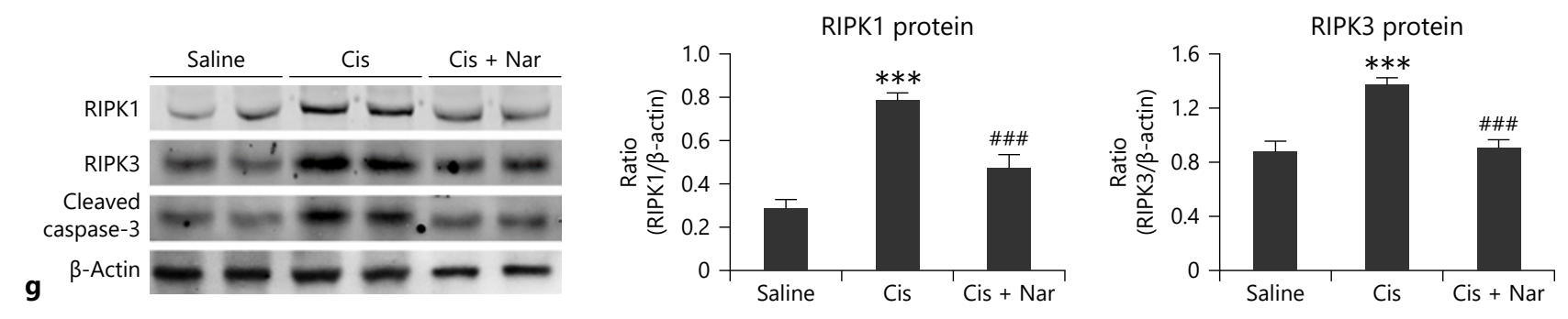

9

(For legend see next pages.) 


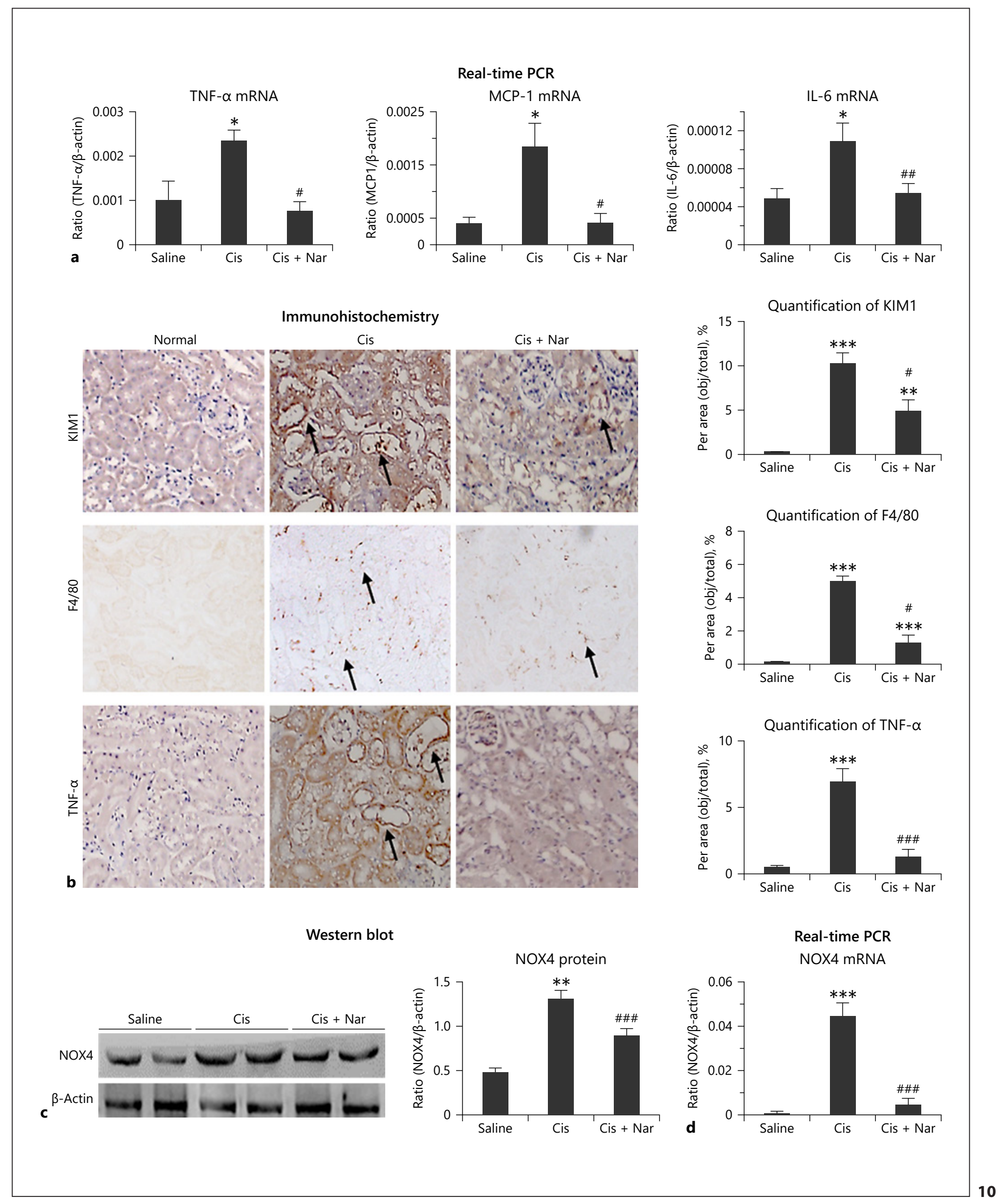

(For legend see next page.) 
$3^{\prime}$ UTR of MLKL, thereby alleviating kidney injury by limiting necroptosis [32]. We also identified that wogonin, a TCM monomer, prevents cisplatin-induced necroptosis and renal injury by binding to the ATPbinding pocket of RIPK1 [28], and treatment with Cpd71 , a novel RIPK1 inhibitor, attenuated cisplatin nephropathy [33]. The results of the current study showed that loss of Smad3 prevented cisplatin-activated necroptotic signaling, with Smad3 deficiency significantly blocked RIPK-mediated programmed cell death. This result indicates Smad3 may play an important role in cell necroptosis. Additionally, we found that disruption or pharmacological inhibition of Smad3 decreased cisplatin-induced inflammatory response both in vivo and in vitro. This finding is consistent with previous reports that global Smad3 KO limited ischemic AKI by downregulating the production of inflammatory indexes such as MCP-1 and IL-6 [21].

Second, we found that Smad3 plays a crucial role in cisplatin-induced renal injury, programmed cell death, and inflammation by limiting NOX4-dependent oxidant stress. Recent studies indicate that diseases caused by cisplatin toxicity are closely correlated with increased ROS levels [34]. The major enzymes involved in ROS production in cisplatin nephrotoxicity are nicotinamide adenine dinucleotide phosphate oxidases, especially NOX4 [35-37]. NOX4, the major NADPH isoform in the kidney, mainly catalyzes reactions that result in the production of $\mathrm{H}_{2} \mathrm{O}_{2}$, which regulates physiological functions [38]. Previous findings indicated that NOX4 contributes to redox processes involved in diabetic nephropathy, AKI, obstructive nephropathy, hypertensive nephropathy, renal cell carcinoma, and other renal dis- eases by activating multiple signaling pathways $[37,39]$. Excessive production of NOX4 promotes cisplatin-induced AKI via ROS-mediated programmed cell death and inflammation [27]. The current study found that NOX4 specifically inhibits the activity of mitochondrial electron transport chain complex I and decreased concentration of complex I subunits, such as NDUFA 9 [40]. In the current study, disruption of Smad3 lowered the cisplatin-induced expression of NOX4, and Smad3 regulated both NOX4 expression and function mainly via 2 mechanisms: (1) interaction with NOX4 to regulate NOX4-dependent ROS production and (2) binding to the promoter region of NOX4 to induce the production of NOX4.

Finally, we designed 2 protocols for Smad3-targeted therapy to investigate the therapeutic potential of Smad3 in the treatment of AKI. Lentivirus-mediated Smad3 KD prevented cisplatin-induced loss of renal function, inflammation, programmed cell death, and oxidative stress in mice. In addition, targeted inhibition of Smad3 by naringenin protects against cisplatin-induced AKI; this finding was consistent with the previous reports that naringenin showed renoprotective effects in response to other stimuli such as gentamicin [41-43].

In conclusion, we found that Smad3 plays a crucial role in cisplatin-induced kidney injury and that Smad3targeted therapy protects against renal injury, programmed cell death, and inflammation through NOX4dependent oxidant stress. Smad3-targeted therapy attenuated cisplatin-induced acute kidney injury. Thus, Smad3 is a promising therapeutic target in AKI.

data of RIPK1, RIPK3, and cleaved caspase-3. Protein levels of KIM1, RIPK1, RIPK3, and cleaved caspase-3 were reduced after treatment with naringenin. Data are mean \pm SEM for 6-8 mice. ${ }^{*} p<0.05,{ }^{* *} p<0.01,{ }^{* * *} p<0.001$ versus control; $\# p<0.01, \# \#<<$

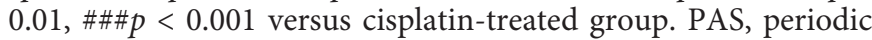
acid-Schiff; HE, hematoxylin-eosin; mTEC: mouse tubular epithelial cells. Nar, naringenin.
Fig. 10. Treatment with naringenin attenuated cisplatin-induced renal inflammation in vivo. a Real-time PCR. Treatment with naringenin reduced mRNA levels of TNF- $\alpha$, IL- 6 , and MCP-1 in vivo. b Immunohistochemistry to detect F4/80+ macrophages, TNF- $\alpha$, and KIM1. Smad3 KD decreased infiltration of F4/80+ macrophages and protein levels of KIM1 and TNF- $\alpha$ in cisplatin ne- phropathy, as the arrows shown in figures. c, d Western blot analysis of NOX4. Naringenin significantly reduced the protein and mRNA levels of NOX4. Data are mean \pm SEM for 6-8 mice.

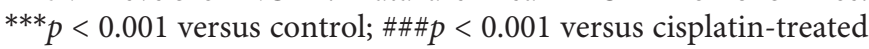
group. IL, interleukin; MCP-1, monocyte chemotactic protein-1; Nar, naringenin. 


\section{Acknowledgements}

The authors thank the Center for Scientific Research of Anhui Medical University for valuable help in our experiment.

\section{Statement of Ethics}

All animal procedures were approved by the Institutional Animal Experimentation Ethics Committee of Anhui Medical University (LLSC20150276). All studies involving animals follow the ARRIVE guidelines.

\section{Conflict of Interest Statement}

The authors have no conflicts of interest to declare.

\section{Funding Sources}

The project was supported by the National Natural Science Foundation of China (No. 81970584 and 81570623 ) and by Science and Technological Fund of Anhui Province for Outstanding Youth of China (No. 1608085J07). These funds are used to buy reagents and laboratory animals.

\section{Author Contributions}

X.-m.M. and H.Y.L. contributed to the experimental design, establishment of Smad3 knockout mouse and AKI model, and manuscript preparation. L.G. and X.w.H. contributed to data analysis and discussion. Q.Y. and J.-n.W. contributed to draft the work and revise it critically for important intellectual content and do experiments. X.-w.H., Y.Z., and Y.-h.D. did experiments and contributed to final approval of the version to be published.

\section{References}

1 Gao L, Wu WF, Dong L, Ren GL, Li HD, Yang $\mathrm{Q}$, et al. Protocatechuic aldehyde attenuates cisplatin-induced acute kidney injury by suppressing Nox-mediated oxidative stress and renal inflammation. Front Pharmacol. 2016;7:479.

2 Gao L, Zhong X, Jin J, Li J, Meng XM. Potential targeted therapy and diagnosis based on novel insight into growth factors, receptors, and downstream effectors in acute kidney injury and acute kidney injury-chronic kidney disease progression. Sig Transduct Target Ther. 2020 Feb 14;5(1):9.

3 Massague J, Wotton D. Transcriptional control by the TGF-beta/Smad signaling system. EMBO J. 2000 Apr 17;19(8):1745-54.

4 Zhang Y, Meng XM, Huang XR, Lan HY. The preventive and therapeutic implication for renal fibrosis by targetting TGF- $\beta / \mathrm{Smad} 3$ signaling. Clin Sci. 2018 Jul 16;132(13):1403-15.

5 Andrade-Silva M, Cenedeze MA, Perandini LA, Felizardo RJF, Watanabe IKM, Agudelo JSH, et al. TLR2 and TLR4 play opposite role in autophagy associated with cisplatin-induced acute kidney injury. Clin Sci. 2018 Aug 31;132(16):1725-39.

6 Lee HT, Chen SW, Doetschman TC, Deng C, D’Agati VD, Kim M. Sevoflurane protects against renal ischemia and reperfusion injury in mice via the transforming growth factorbetal pathway. Am J Physiol Renal Physiol. 2008 Jul;295(1):F128-36.

7 Guan Q, Nguan CY, Du C. Expression of transforming growth factor-betal limits renal ischemia-reperfusion injury. Transplantation. 2010 Jun 15;89(11):1320-7.

8 Huynh $\mathrm{P}$, Chai Z. Transforming growth factor beta (TGFbeta) and related molecules in chronic kidney disease (CKD). Clin Sci. 2019 Jan 31;133(2):287-313.

9 Gewin L, Vadivelu S, Neelisetty S, Srichai MB, Paueksakon P, Pozzi A, et al. Deleting the
TGF- $\beta$ receptor attenuates acute proximal tubule injury. J Am Soc Nephrol. 2012 Dec; 23(12):2001-11.

10 Gentle ME, Shi S, Daehn I, Zhang T, Qi H, Yu $\mathrm{L}$, et al. Epithelial cell TGF $\beta$ signaling induces acute tubular injury and interstitial inflammation. J Am Soc Nephrol. 2013 Apr;24(5): 787-99.

11 Jeong BY, Park SR, Cho S, Yu SL, Lee HY, Park CG, et al. TGF- $\beta$-mediated NADPH oxidase 4-dependent oxidative stress promotes colistin-induced acute kidney injury. J Antimicrob Chemother. 2018 Apr 1;73(4):962-72.

12 Meng XM, Huang XR, Chung AC, Qin W, Shao X, Igarashi P, et al. Smad2 protects against TGF-beta/Smad3-mediated renal fibrosis. J Am Soc Nephrol. 2010 Sep;21(9): 1477-87.

13 Meng XM, Tang PM, Li J, Lan HY. TGF- $\beta$ / Smad signaling in renal fibrosis. Front Physiol. 2015;6:82.

14 Meng XM, Nikolic-Paterson DJ, Lan HY. TGF-beta: the master regulator of fibrosis. Nat Rev Nephrol. 2016 Jun;12(6):325-38.

15 Kilari S, Yang B, Sharma A, McCall DL, Misra $S$. Increased transforming growth factor beta (TGF-beta) and pSMAD3 signaling in a $\mathrm{Mu}$ rine Model for Contrast Induced Kidney Injury. Sci Rep. 2018 Apr 26;8(1):6630.

16 Sato M, Muragaki Y, Saika S, Roberts AB, Ooshima A. Targeted disruption of TGF-beta1/Smad3 signaling protects against renal tubulointerstitial fibrosis induced by unilateral ureteral obstruction. J Clin Invest. 2003 Nov; 112(10):1486-94.

17 Yang Q, Ren G-l., Wei B, Jin J, Huang XR, Shao W, et al. Conditional knockout of TGF$\beta R I I / S m a d 2$ signals protects against acute renal injury by alleviating cell necroptosis, apoptosis and inflammation. Theranostics. 2019;9(26):8277-93.
18 Ghayur A, Padwal MK, Liu L, Zhang J, Margetts PJ. SMAD3-dependent and -independent pathways in glomerular injury associated with experimental glomerulonephritis. Am J Physiol Renal Physiol. 2019 Jul 1;317(1):F152-62.

19 Xu BH, Sheng J, You YK, Huang XR, Ma RCW, Wang Q, et al. Deletion of Smad3 prevents renal fibrosis and inflammation in type 2 diabetic nephropathy. Metabolism. 2020 Feb;103:154013.

20 Jiang M, Fan J, Qu X, Li S, Nilsson SK, Sun YBY, et al. Combined blockade of $S \operatorname{mad} 3$ and JNK pathways ameliorates progressive fibrosis in folic acid nephropathy. Front Pharmacol. 2019;10:880.

21 Nath KA, Croatt AJ, Warner GM, Grande JP. Genetic deficiency of Smad3 protects against murine ischemic acute kidney injury. Am J Physiol Renal Physiol. 2011 Aug;301(2): F436-42.

22 Lai W, Tang Y, Huang XR, Ming-Kuen Tang $\mathrm{P}, \mathrm{Xu} A$, Szalai AJ, et al. C-reactive protein promotes acute kidney injury via Smad3-dependent inhibition of CDK2/cyclin E. Kidney Int. 2016 Sep;90(3):610-26.

23 Wang JN, Yang Q, Yang C, Cai YT, Xing T, Gao L, et al. Smad3 promotes AKI sensitivity in diabetic mice via interaction with p53 and induction of NOX4-dependent ROS production. Redox Biol. 2020 Feb 26;32:101479.

24 Liu X, Wang W, Hu H, Tang N, Zhang C, Liang $\mathrm{W}$, et al. Smad3 specific inhibitor, naringenin, decreases the expression of extracellular matrix induced by TGF-betal in cultured rat hepatic stellate cells. Pharm Res. 2006 Jan;23(1):82-9.

25 Lou C, Zhang F, Yang M, Zhao J, Zeng W, Fang $X$, et al. Naringenin decreases invasiveness and metastasis by inhibiting TGF- $\beta$ induced epithelial to mesenchymal transition in pancreatic cancer cells. PLoS One. 2012; 7(12):e50956. 
26 Meng XM, Zhang Y, Huang XR, Ren GL, Li J, Lan HY. Treatment of renal fibrosis by rebalancing TGF- $\beta /$ Smad signaling with the combination of asiatic acid and naringenin. Oncotarget. 2015 Nov 10;6(35):36984-97.

27 Meng XM, Ren GL, Gao L, Yang Q, Li HD, $\mathrm{Wu} \mathrm{WF}$, et al. NADPH oxidase 4 promotes cisplatin-induced acute kidney injury via ROS-mediated programmed cell death and inflammation. Lab Invest. 2018 Jan;98(1):6378.

28 Meng XM, Li HD, Wu WF, Ming-Kuen Tang $\mathrm{P}$, Ren GL, Gao L, et al. Wogonin protects against cisplatin-induced acute kidney injury by targeting RIPK1-mediated necroptosis. Lab Invest. 2018 Jan;98(1):79-94.

29 Gao L, Liu M-M, Zang H-m., Ma Q-Y, Yang $\mathrm{Q}$, Jiang $\mathrm{L}$, et al. Restoration of E-cadherin by PPBICA protects against cisplatin-induced acute kidney injury by attenuating inflammation and programmed cell death. Lab Invest. 2018 Jul;98(7):911-23.

30 Linkermann A, Bräsen $\mathrm{JH}$, Darding $\mathrm{M}$, Jin MK, Sanz AB, Heller JO, et al. Two independent pathways of regulated necrosis mediate ischemia-reperfusion injury. Proc Natl Acad Sci U S A. 2013 Jul 16;110(29):12024-9.

31 Xu Y, Ma H, Shao J, Wu J, Zhou L, Zhang Z, et al. A role for tubular necroptosis in cisplatin-induced AKI. J Am Soc Nephrol. 2015 Nov;26(11):2647-58.
32 Jiang L, Liu XQ, Ma Q, Yang Q, Gao L, Li HD, et al. hsa-miR-500a-3P alleviates kidney injury by targeting MLKL-mediated necroptosis in renal epithelial cells. FASEB J. 2019 Mar; 33(3):3523-35.

33 Wang JN, Liu MM, Wang F, Wei B, Yang Q, Cai YT, et al. RIPK1 inhibitor Cpd-71 attenuates renal dysfunction in cisplatin-treated mice via attenuating necroptosis, inflammation and oxidative stress. Clin Sci. 2019 Jul 31; 133(14):1609-27.

34 Kaur T, Borse V, Sheth S, Sheehan K, Ghosh $S$, Tupal S, et al. Adenosine A1 receptor protects against cisplatin ototoxicity by suppressing the NOX3/STAT1 inflammatory pathway in the cochlea. J Neurosci. 2016 Apr 6;36(14): 3962-77.

35 Sedeek M, Nasrallah R, Touyz RM, Hébert RL. NADPH oxidases, reactive oxygen species, and the kidney: friend and foe. J Am Soc Nephrol. 2013 Oct;24(10):1512-8.

36 Wang Y, Luo X, Pan H, Huang W, Wang X, Wen $\mathrm{H}$, et al. Pharmacological inhibition of $\mathrm{NADPH}$ oxidase protects against cisplatin induced nephrotoxicity in mice by two step mechanism. Food Chem Toxicol. 2015 Sep; 83:251-60.

37 Yang Q, Wu FR, Wang JN, Gao L, Jiang L, Li $\mathrm{HD}$, et al. Nox4 in renal diseases: an update. Free Radic Biol Med. 2018 Jun 30;124:466-72.
38 Okamura DM, Pennathur S. The balance of powers: redox regulation of fibrogenic pathways in kidney injury. Redox Biol. 2015 Dec; 6:495-504.

39 Shen CJ, Chang KY, Lin BW, Lin WT, Su CM, Tsai JP, et al. Oleic acid-induced NOX4 is dependent on ANGPTL4 expression to promote human colorectal cancer metastasis. Theranostics. 2020;10(16):7083-99.

40 Koziel R, Pircher H, Kratochwil M, Lener B, Hermann M, Dencher NA, et al. Mitochondrial respiratory chain complex I is inactivated by NADPH oxidase NOX4. Biochem J. 2013 Jun 1;452(2):231-9.

41 Fouad AA, Albuali WH, Zahran A, Gomaa W. Protective effect of naringenin against gentamicin-induced nephrotoxicity in rats. Environ Toxicol Pharmacol. 2014 Sep;38(2): 420-9.

42 Khan TH, Ganaie MA, Alharthy KM, Madkhali H, Jan BL, Sheikh IA. Naringenin prevents doxorubicin-induced toxicity in kidney tissues by regulating the oxidative and inflammatory insult in Wistar rats. Arch Physiol Biochem. 2020 Oct;126(4):300-7.

$43 \mathrm{Mu} \mathrm{L}, \mathrm{Hu}$ G, Liu J, Chen Y, Cui W, Qiao L. Protective effects of naringenin in a rat model of sepsis-triggered acute kidney injury via activation of antioxidant enzymes and reduction in urinary angiotensinogen. Med Sci Monit. 2019 Aug 11;25:5986-91. 